\title{
Neutron-mirror neutron mixing and neutron stars
}

\author{
Zurab Berezhiani ${ }^{1,2, a}$, Riccardo Biondi ${ }^{2}$, Massimo Mannarelli ${ }^{2}$, Francesco Tonelli ${ }^{3}$ \\ ${ }^{1}$ Dipartimento di Fisica e Chimica, Università di L'Aquila, 67100 Coppito, L'Aquila, Italy \\ 2 INFN, Laboratori Nazionali del Gran Sasso, 67010 Assergi, L'Aquila, Italy \\ ${ }^{3}$ Consiglio Nazionale delle Ricerche, CNR-SPIN, 67100 Coppito, L'Aquila, Italy
}

Received: 22 April 2021 / Accepted: 28 September 2021 / Published online: 26 November 2021

(C) The Author(s) 2021

\begin{abstract}
The oscillation of neutron $n$ into mirror neutron $n^{\prime}$, its mass degenerate partner from dark mirror sector, can gradually transform the neutron stars into the mixed stars consisting in part of mirror dark matter. In quark stars $n-n^{\prime}$ transitions are suppressed. We study the structure of mixed stars and derive the mass-radius scaling relations between the configurations of purely neutron star and maximally mixed star (MMS) containing equal amounts of ordinary and mirror components. In particular, we show that the MMS masses can be at most $M_{N S}^{\max } / \sqrt{2}$, where $M_{N S}^{\max }$ is a maximum mass of a pure neutron star allowed by a given equation of state. We evaluate $n-n^{\prime}$ transition rate in neutron stars, and show that various astrophysical limits on pulsar properties exclude the transition times in a wide range $10^{5}$ year $<\tau_{\varepsilon}<10^{15}$ year. For short transition times, $\tau_{\varepsilon}<10^{5}$ year, the different mixed stars of the same mass can have different radii, depending on their age, which possibility can be tested by the NICER measurements. We also discuss subtleties related with the possible existence of mixed quark stars, and possible implications for the gravitational waves from the neutron star mergers and associated electromagnetic signals.
\end{abstract}

\section{Introduction}

The idea that there may exist a hidden particle sector consisting of mirror duplicates of the observed standard particles was introduced long time ago for restoring parity as a fundamental symmetry [1-3]. In this context, all known particles: electron $e$, proton $p$, neutron $n$, neutrinos $v$ etc. must have their mirror partners $e^{\prime}, p^{\prime}, n^{\prime}, v^{\prime}$ etc. which are supposed to be sterile to our Standard Model (SM) interactions $S U(3) \times S U(2) \times U(1)$, but have their own $S U(3)^{\prime} \times S U(2)^{\prime} \times U(1)^{\prime}$ gauge interactions (see Refs. [46] for reviews and [7] for a historical overview).

\footnotetext{
a e-mail: zurab.berezhiani@1ngs.infn.it (corresponding author)
}

More generically, one can consider theories based on the direct product $G \times G^{\prime}$ of two identical gauge factors (SM or some its extension), with the Lagrangian

$\mathcal{L}_{\text {tot }}=\mathcal{L}+\mathcal{L}^{\prime}+\mathcal{L}_{\text {mix }}$

where $\mathcal{L}$ describes ordinary (O) particles, $\mathcal{L}^{\prime}$ describes their mirror $(\mathrm{M})$ partners and $\mathcal{L}_{\text {mix }}$ stands for possible crossinteractions between the $\mathrm{O}$ and $\mathrm{M}$ particles. The identical forms of the Lagrangians $\mathcal{L}$ and $\mathcal{L}^{\prime}$ is ensured by $Z_{2}$ symmetry under the exchange $G \leftrightarrow G^{\prime}$ of all O particles (fermions, Higgs and gauge fields) with their $\mathrm{M}$ twins ('primed' fermions, Higgs and gauge fields). In view of chiral character of the SM, this discrete symmetry can be imposed with or without chirality change between $\mathrm{O}$ and $\mathrm{M}$ fermions. Mirror parity corresponds to the former possibility, however this difference will not be important for our further considerations. If mirror $Z_{2}$ parity is unbroken, then $O$ particles and their $\mathrm{M}$ partners are degenerate in mass, and the interaction terms in $\mathcal{L}$ and $\mathcal{L}^{\prime}$ are exactly identical [2,3]. Also the case of spontaneously broken $Z_{2}$ symmetry has been considered in the literature $[8,9]$ in which case the weak scales in two sectors can be different, and thus $\mathrm{M}$ particles and their $\mathrm{O}$ partners are no more mass degenerate. In particular, this occurs in the mirror twin Higgs scenarios [5,10].

Mirror matter, gravitationally coupled to ordinary matter but dark in terms of our photons, could represent the part or even entire amount of dark matter in the Universe, with the abundance related to the mirror baryon asymmetry. The collisional and dissipative properties of $\mathrm{M}$ baryons/nuclei/atoms can have specific implications for cosmology [11-17] as e.g. existence of M stars which can be detected as Machos of different masses [18].

Lagrangian $\mathcal{L}_{\text {mix }}$ in (1) may contain cross-terms that induce oscillation phenomena between $\mathrm{O}$ and $\mathrm{M}$ sectors. In fact, any neutral $\mathrm{O}$ particle, elementary (e.g. neutrinos) or composite (e.g. neutrons) can have mixing with its $\mathrm{M}$ twin and oscillate into the latter. For example, the photon 
kinetic mixing with mirror photon [19] induces the positronium oscillation into mirror positronium [20]. Oscillation between the $\mathrm{O}$ and $\mathrm{M}$ neutral mesons, or between the muonium and mirror muonium, can be induced by the gauge bosons of a flavor symmetry interacting with the fermions of both sectors [21,22].

Most interesting are the cross-interactions which violate the lepton and/or baryon numbers of both sectors. Namely, "active-sterile" mixing between ordinary $v_{e, \mu, \tau}$ and mirror $v_{e, \mu, \tau}^{\prime}$ neutrinos can be induced via effective operators $\frac{1}{M} \ell \ell^{\prime} \phi \phi^{\prime}+$ h.c. with a large cutoff scale $M$, where $\ell$ and $\phi$ are the lepton and Higgs doublets of $\mathrm{O}$ sector and $\ell^{\prime}$ and $\phi^{\prime}$ are their M partners [23-27]. These operators, violating B - L as well as $\mathrm{B}^{\prime}-\mathrm{L}^{\prime}$, also suggest a co-leptogenesis mechanism [28-30] which creates comparable baryon asymmetries in both $\mathrm{O}$ and $\mathrm{M}$ worlds, and can explain the relation $\Omega_{\mathrm{B}}^{\prime} \geq \Omega_{\mathrm{B}}$ between the dark matter and baryon fractions in the Universe [31,32].

In this paper we shall concentrate on the mixing between the neutron $n$ and its mirror partner $n^{\prime}[33,34]$ :

$\varepsilon \overline{n^{\prime}} n+$ h.c.

In fact, the mixed Lagrangian $\mathcal{L}_{\text {mix }}$ may include effective operators involving color-singlet combinations of ordinary $u, d$ and mirror $u^{\prime}, d^{\prime}$ quarks:

$\frac{1}{\mathcal{M}^{5}}\left(\overline{u^{\prime}} \overline{d^{\prime}} \overline{d^{\prime}}\right)(u d d)+$ h.c.,

(the gauge and Lorentz indices are omitted). These operators violate both $\mathrm{O}$ and $\mathrm{M}$ baryon numbers by one unit, $\Delta \mathrm{B}=1$ and $\Delta \mathrm{B}^{\prime}=-1$, but the overall baryon number $\overline{\mathrm{B}}=\mathrm{B}+\mathrm{B}^{\prime}$ is conserved. In UV complete theories they can be induced via a see-saw like mechanism involving new heavy particles, colored scalars and neutral fermions, with masses $\sim \mathcal{M}[33$, 35]. Hence, for $\mathcal{M}$ at few $\mathrm{TeV}$, the underlying theories can be testable at the LHC and future accelerators $[35,36]$.

Operators (3) induce $n-n^{\prime}$ mass mixing (2) with

$\varepsilon=\frac{C^{2} \Lambda_{\mathrm{QCD}}^{6}}{\mathcal{M}^{5}}=C^{2}\left(\frac{10 \mathrm{TeV}}{\mathcal{M}}\right)^{5} \times 10^{-15} \mathrm{eV}$

where $C=O(1)$ is the operator dependent numerical factor in the determination of the matrix element $\langle 0|u d d| n\rangle$.

The phenomenon of $n-n^{\prime}$ oscillation is analogous to that of neutron-antineutron $(n-\bar{n})$ oscillation $[37,38]$ (for a review, see [39-41]), and in fact both phenomena can be related to the same new physics $[33,35]$. However, $n-\bar{n}$ oscillation is severely restricted by experiment. Namely, the direct experimental limit on $n-\bar{n}$ oscillation is $\varepsilon_{n \bar{n}}<7.7 \times$ $10^{-24} \mathrm{eV}$ while the nuclear stability bounds are yet stronger, $\varepsilon_{n \bar{n}}<2.5 \times 10^{-24} \mathrm{eV}$ or so [39]. As for $n-n^{\prime}$ oscillation, it is kinematically forbidden for neutrons bound in nuclei, simply by energy conservation [33], and so nuclear stability imposes no limit on $n-n^{\prime}$ mixing. But for free neutrons it can be effective, and may be even much faster than the neutron decay $[33,34]$.

Namely, $n-n^{\prime}$ mixing can be as large as $\varepsilon \sim 10^{-15} \mathrm{eV}$, corresponding to the characteristic time $\tau_{n n^{\prime}}=\varepsilon^{-1} \sim 1 \mathrm{~s}$ or even smaller. This possibility is not excluded by existing astrophysical and cosmological limits [33], and it can have observable effects for the ultra-high energy cosmic rays $[43,44]$, for neutrons from the solar flares [45] and for primordial nucleosynthesis [46]. Its search via the neutron disappearance $\left(n \rightarrow n^{\prime}\right)$ and regeneration $\left(n \rightarrow n^{\prime} \rightarrow n\right)$ experiments can be perfectly accessible at existing neutron facilities $[33,47,48]$.

In normal experimental conditions $n-n^{\prime}$ oscillation is suppressed by environmental effects as e.g. mirror magnetic fields at the Earth [34]. This can be the reason why the disappearance of neutrons within few seconds so far skipped the experimental detection. Several dedicated experiments were performed searching $n-n^{\prime}$ oscillations [49-55] in controlled background conditions and they still do not exclude small $\tau_{n n^{\prime}}$. Moreover, some of these experiments show anomalous deviations from null hypothesis indicating to $\tau_{n n^{\prime}} \sim 10 \mathrm{~s}$ or so $[54,56]$. New experiments for testing these effects are underway [57-59].

Larger values of $\varepsilon$ are also allowed if $n$ and $n^{\prime}$ are not exactly mass-degenerate. Moreover, $n-n^{\prime}$ oscillation with $\varepsilon \sim 10^{-10} \mathrm{eV}$ or so can solve the neutron lifetime problem, the $4 \sigma$ discrepancy between the neutron lifetimes measured via the bottle and beam experiments, provided that $n$ and $n^{\prime}$ have a mass splitting $m_{n^{\prime}}-m_{n} \sim 100 \mathrm{neV}$ [60]. Such a small splitting can be naturally realized in models in which $Z_{2}$ parity is spontaneously broken $[8,9]$ but with a rather small difference between the $\mathrm{O}$ and M Higgs VEVs $\langle\phi\rangle$ and $\left\langle\phi^{\prime}\right\rangle[61]$.

Although $n-n^{\prime}$ transition is suppressed for neutrons bound in nuclei by nuclear forces, it can be effective in neutron stars (NS) where the neutrons are bound by gravity. The transformation of neutrons into mirror neutrons should decrease the degeneracy pressure, thereby softening the equation of state (EoS) of the system. The gravitational binding energy increases and thus the process is energetically favored. In this way, a NS born after supernova explosions should gradually evolve in a mixed star (MS) partially consisting of mirror matter, and asymptotically in time could even reach the maximally mixed star (MMS) configuration with equal amounts of $\mathrm{O}$ and $\mathrm{M}$ components.

In the present paper we shall concentrate on the implications of $n-n^{\prime}$ conversion for the NS. As was already noted in [33], for $\varepsilon>10^{-15} \mathrm{eV}$ (i.e. $\tau_{n n^{\prime}}>1 \mathrm{~s}$ or so) the transformation time is several orders of magnitude larger than the age of the Universe. Nevertheless, the NS transformation into the MS with small mirror cores can have astrophysical signatures for the pulsar dynamics and for the NS gravitational merg- 
ers. Some respective implications were recently addressed in [62-67].

Here we estimate the $n-n^{\prime}$ conversion time $\tau_{\varepsilon}$ for the NS and show that the range $\tau_{\varepsilon}<10^{15}$ year is disfavored by the limits from the pulsar evolution dynamics, especially by their heating due to $n-n^{\prime}$ conversion. However, also rather small values $\tau_{\varepsilon}<10^{5}$ year can be allowed in which case the older NS with the masses below some (EoS dependent) critical threshold should be already transformed into the MMS. As for the NS with the masses exceeding the threshold value, $n-n^{\prime}$ conversion produces a gravitationally unstable MS that collapses to a black hole $(\mathrm{BH})$. However, one cannot exclude that the heavier compact objects are not the NS but they are represented by quark or hybrid stars for which $n-n^{\prime}$ mixing has quite different implications.

Our work is organized as follows. In Sect. 2 we discuss effects of $n-n^{\prime}$ mixing on the evolution and structure of mixed stars, and derive the mass-radius scaling relations between the NS and MMS. In Sect. 3 we discuss $n-n^{\prime}$ oscillations in dense nuclear matter and estimate $n-n^{\prime}$ transformation time of neutron stars. In Sect. 4 we discuss possible effects for the pulsar observations, and in Sect. 5 for the neutron star coalescences and associated signals. We draw our conclusions in Sect. 6.

\section{Neutron star evolution into mixed stars}

Neutron stars are presumably born after the supernova explosions of massive stars. They are believed to have an onion-like structure that schematically consists of a crust dominated by nuclei and a liquid nuclear matter in the core whose dominant component are neutrons, with some fractions of protons, electrons, muons and perhaps of heavier baryons too (for reviews, see e.g. [68,69]).

The supernova core-collapse should produce a NS consisting of ordinary nuclear matter. But then $n \rightarrow n^{\prime}$ transitions will start to produce $M$ neutrons in its interior and the original NS will gradually transform into a MS, with the mirror matter fraction increasing in time. We consider that this process is rather slow, with the effective $n \rightarrow n^{\prime}$ transformation rate being being less than the star cooling rate. Under these circumstances the evolution should be adiabatic and it can be described by the Boltzmann equations

$\frac{d N_{\mathrm{O}}}{d t}=-\Gamma N_{\mathrm{O}}+\Gamma^{\prime} N_{\mathrm{M}}, \quad \frac{d N_{\mathrm{M}}}{d t}=\Gamma N_{\mathrm{O}}-\Gamma^{\prime} N_{\mathrm{M}}$

where $N_{\mathrm{O}}(t)$ and $N_{\mathrm{M}}(t)$ respectively are the amounts of $\mathrm{O}$ and $\mathrm{M}$ baryons in the star at the time $t$ and $\Gamma$ is the $n \rightarrow n^{\prime}$ conversion rate (to be estimated in next section), whereas $\Gamma^{\prime} \ll \Gamma$ is the rate of the inverse process $n^{\prime} \rightarrow n$. Starting from a newborn NS with $N_{\mathrm{M}}=0$ and $N_{\mathrm{O}}=N_{0}$ at $t=0$, $N_{\mathrm{M}}(t)$ should increase and $N_{\mathrm{O}}(t)$ will decrease in time. But the overall amount of baryons remains constant, $N_{\mathrm{O}}(t)+$ $N_{\mathrm{M}}(t)=N_{0}$, since $n-n^{\prime}$ oscillation conserves the combined baryon number $\overline{\mathrm{B}}=\mathrm{B}+\mathrm{B}^{\prime} .1$

Then, neglecting the inverse reaction rate $\Gamma^{\prime}$, Eq. (5) reduce to a single equation

$\frac{d X}{d t}=\Gamma(1-X)$

where $X(t)=N_{\mathrm{M}}(t) / N_{0}$ is the fraction of $\mathrm{M}$ baryons at the time $t$ while $N_{\mathrm{O}}(t) / N_{0}=1-X(t)$, and as initial condition we have $X=0$ at $t=0$. The transition rate $\Gamma=\Gamma(X)$ is not constant: it depends on $X$ which in itself evolves in time. It can be presented as

$\Gamma(X)=\Gamma_{0} \mathcal{F}(X)$

where $\Gamma_{0}=(d X / d t)_{t=0}$ is the 'starting' rate of conversion for a given star, and the function $\mathcal{F}(X)$ normalized as $\mathcal{F}(0)=$ 1 , comprises the dependence on the mirror admixture. Then, by integrating Eq. (6), we obtain the MS age $t$ as function of mirror fraction $X$ in its interior:

$t(X)=\int_{0}^{X} \frac{d x}{\Gamma(x)(1-x)}=\tau_{\varepsilon} \int_{0}^{X} \frac{d x}{(1-x) \mathcal{F}(x)}$

where the characteristic transition time $\tau_{\varepsilon}=\Gamma_{0}^{-1}$ will depends on $\varepsilon$ as $\tau_{\varepsilon} \propto 1 / \varepsilon^{2}$. Since $\mathcal{F} \rightarrow 1$ at $x \rightarrow 0$, for $X \ll 1$ we have

$\dot{X}=1 / \tau_{\varepsilon} \quad \longrightarrow \quad X=t / \tau_{\varepsilon}$

meaning that in the younger MS the mirror fraction $X$ increases linearly with time $t$ until $t \ll \tau_{\varepsilon}$. But with $X$ growing the evolution should gradually slow down.

Asymptotically in time the star can evolve to the final equilibrium configuration with $X=1 / 2$ (i.e. to the MMS with equal amounts of the $\mathrm{O}$ and $\mathrm{M}$ components), provided that it remains stable during the evolution. The latter depends on it total baryon number $N_{0}$. The initial NS can be stable only if $N_{0}<N_{\mathrm{NS}}^{\max }$, where $N_{\mathrm{NS}}^{\max }$ is the maximal baryon number which corresponds to the NS maximal mass $M_{\mathrm{NS}}^{\mathrm{max}}$ affordable by the given EoS. As we show in next section, only the stars with $N_{0}<N_{\mathrm{NS}}^{\max } / \sqrt{2}$ can become the MMS, with final masses $M<M_{\mathrm{NS}}^{\max } / \sqrt{2}$. The stars with $N_{0}>N_{\mathrm{NS}}^{\max } / \sqrt{2}$ should instead collapse at the stage $t_{\text {coll }}$ when their mirror fraction reaches certain $\left(N_{0}\right.$-dependent) value $X_{\text {coll }}\left(N_{0}\right)<1 / 2$. In this case Eq. (8), integrated up to $X=X_{\text {coll }}$, will define the MS lifetime $t_{\text {coll }}=t\left(X_{\text {coll }}\right)$.

\footnotetext{
${ }^{1}$ In principle, the neutron could have mixings both with the mirror neutron $n^{\prime}$ and mirror antineutron $\bar{n}^{\prime}$ [70]. In this case $\bar{B}$ would not be conserved, but here we do not consider this possibility which will be discussed elsewhere.
} 


\subsection{Structure of mixed neutron stars}

As far as the evolution is adiabatic, we can use a static "two fluid" description in which the total energy density and pressure can be decomposed as the sum of ordinary and mirror components, i.e. $\rho=\rho_{\mathrm{O}}+\rho_{\mathrm{M}}$ and $p=p_{\mathrm{O}}+p_{\mathrm{M}}$. Thus, the total energy-momentum tensor $T_{v}^{\mu}=\operatorname{diag}(\rho,-p,-p,-p)$ can be split as $T_{\mu \nu}=T_{\mu \nu}^{\mathrm{O}}+T_{\mu \nu}^{\mathrm{M}}$, where $T_{\mu \nu}^{\mathrm{O}}$ and $T_{\mu \nu}^{\mathrm{M}}$ respectively are the energy-momentum tensors of $\mathrm{O}$ and $\mathrm{M}$ components.

Assuming spherical symmetry, at any moment of time the MS can be described as a static configuration of two concentric $\mathrm{O}$ and $\mathrm{M}$ spheres respectively with the radii $R_{\mathrm{O}} \equiv R$ and $R_{\mathrm{M}} \leq R$ corresponding to the positions where $p_{\mathrm{O}}(r)$ and $p_{\mathrm{M}}(r)$ vanish. Hence, we take the metric tensor of the standard spherically symmetric form, $g_{\mu v}=$ $\operatorname{diag}\left(-g_{t t}, g_{r r}, r^{2}, r^{2} \sin ^{2} \theta\right)$, where

$g_{t t}(r)=\exp [2 \phi(r)], \quad g_{r r}(r)=\frac{1}{1-2 m(r) / r}$

Here $m(r)$ is the total gravitational mass within the radius $r$, and $\phi(r)$ is the gravitational potential. (In this section we use geometrized units, $c=1$ and $G=1$.)

In hydrostatic equilibrium the density and pressure profiles in the star are determined by the Tolman-OpenheimerVolkoff (TOV) equations [71,72]:

$$
\begin{aligned}
\frac{d m}{d r} & =4 \pi \rho r^{2} \\
\frac{d p}{d r} & =-(\rho+p) \frac{d \phi}{d r} \\
\frac{1}{\rho+p} \frac{d p}{d r} & =\frac{m+4 \pi p r^{3}}{2 m r-r^{2}}
\end{aligned}
$$

The first differential equation above is linear, and we can split it between two components, $m(r)=m_{\mathrm{O}}(r)+m_{\mathrm{M}}(r)$ :

$\frac{d m_{\mathrm{O}}}{d r}=4 \pi \rho_{\mathrm{O}} r^{2}, \quad \frac{d m_{\mathrm{M}}}{d r}=4 \pi \rho_{\mathrm{M}} r^{2}$

where $m_{\alpha}(r)=4 \pi \int_{0}^{r} \rho_{\alpha}(r) r^{2} d r(\alpha=\mathrm{O}, \mathrm{M})$. Therefore, the total gravitational mass of the MS is given by the sum $M_{\mathrm{O}}+M_{\mathrm{M}}=M=m(R)$. Since both components are in hydrostatic equilibrium, $\dot{\rho}_{\alpha}, \dot{p}_{\alpha}=0$, the continuity equations for each energy-momentum tensor separately, $\nabla_{\mu} T_{v}^{\mu(\alpha)}=0$, give

$$
-\partial_{r} \phi=\frac{\partial_{r} p_{\mathrm{O}}}{\rho_{\mathrm{O}}+p_{\mathrm{O}}}=\frac{\partial_{r} p_{\mathrm{M}}}{\rho_{\mathrm{M}}+p_{\mathrm{M}}}=\frac{\partial_{r} p}{\rho+p}
$$

where the last equality follows from the first two. At the boundary $r=R$ one has $\exp [2 \phi(R)]=1-2 M / R$.

As for Eq. (13), it in fact couples the pressures and energy densities of the two fluids. Using Eqs. (14) and (15), it gives the coupled differential equations for the $\mathrm{O}$ and $\mathrm{M}$ components:

$$
\frac{\partial_{r} p_{\mathrm{O}}}{\rho_{\mathrm{O}}+p_{\mathrm{O}}}=\frac{\partial_{r} p_{\mathrm{M}}}{\rho_{\mathrm{M}}+p_{\mathrm{M}}}=\frac{m_{\mathrm{M}}+m_{\mathrm{O}}+4 \pi\left(p_{\mathrm{M}}+p_{\mathrm{O}}\right) r^{3}}{2\left(m_{\mathrm{M}}+m_{\mathrm{O}}\right) r-r^{2}}
$$

The above system can be solved once the EoS are given, and the appropriate boundary conditions are chosen. In our case, both components $\alpha=\mathrm{O}, \mathrm{M}$ should have the same EoS $p_{\alpha}=F\left(\rho_{\alpha}\right)$ by mirror symmetry. Then one can find their density profiles $\rho_{\alpha}(r)$ by fixing the respective central densities, $\rho_{\mathrm{O}}(0)=\rho_{\mathrm{cO}}$ and $\rho_{\mathrm{M}}(0)=\rho_{\mathrm{cM}}$. Once the density profiles is found, the gravitational potential $\phi(r)$ can be obtained directly from Eq. (15).

For initial configuration of NS composed exclusively of O baryons, the system of Eqs. (14) and (16) reduces to the standard one-component TOV equations:

$$
\frac{d m_{\mathrm{O}}}{d r}=4 \pi \rho_{\mathrm{O}} r^{2}, \quad \frac{1}{\rho_{\mathrm{O}}+p_{\mathrm{O}}} \frac{d p_{\mathrm{O}}}{d r}=\frac{m_{\mathrm{O}}+4 \pi p_{\mathrm{O}} r^{3}}{2 m_{\mathrm{O}} r-r^{2}}
$$

Solving these equations with a given central density $\rho_{\mathrm{O}}(0)=$ $\rho_{c}$, one can find the NS density profile:

$\rho(r)=\rho_{\mathrm{O}}(r)=\rho_{c} f(r)$

where the function $f(r)$ is normalized as $f(0)=1$ and its shape depends on the EoS. The NS radius $R_{\mathrm{NS}} \equiv R_{\mathrm{O}}$ corresponds to the distance at which $f(r)$ vanishes, while the gravitational mass is

$M_{\mathrm{NS}}\left(\rho_{\mathrm{c}}\right)=4 \pi \int_{0}^{R_{\mathrm{NS}}} \rho(r) r^{2} d r=4 \pi \rho_{c} \int_{0}^{\infty} f(r) r^{2} d r$

In fact, the integration can be extended to infinity since $f(r)=0$ for $r>R_{\mathrm{NS}}$.

An important feature is that, for any given EoS, there is no gravitationally stable solution if $\rho_{\mathrm{c}}$ is larger than a certain critical value $\rho_{c}^{\max }$. This determines the NS maximum mass $M_{\mathrm{NS}}^{\max }=M_{\mathrm{NS}}\left(\rho_{c}^{\max }\right)$, also called the last stable configuration for a given EoS. Discovery of pulsars with gravitational masses $M \gtrsim 2 M_{\odot}$ [73-76] challenged several EoSs, and excluded the too soft ones. In this respect, as examples we consider two possible EoS which allow sufficiently large maximum masses.

One is the realistic SLy (Skyrme-Lyon) EoS [77], which gives both the energy density and pressure explicitly as functions of the baryon number density $n_{\mathrm{O}}$. The last stable configuration associated to this EoS has a mass $M_{\mathrm{NS}}^{\max } \simeq 2.05 M_{\odot}$ (with the respective baryon number $N_{\mathrm{NS}}^{\max }=2.91 \times 10^{57}$ ), and it corresponds to $\rho_{c}^{\max }=2.86 \times 10^{15} \mathrm{~g} / \mathrm{cm}^{3}\left(n_{\mathrm{c}}^{\max }=\right.$ $1.28 \mathrm{fm}^{-3}$ ) [77].

As a second example we choose two joined polytropes:

$p= \begin{cases}K_{1} \rho^{\gamma_{1}} & \text { for } \rho>\rho_{\mathrm{tr}} \\ K_{2} \rho^{\gamma_{2}} & \text { for } \rho<\rho_{\mathrm{tr}}\end{cases}$ 
with $\gamma_{1}=3$ for the inner part of the NS and $\gamma_{2}=4 / 3$ for its outer part, with transition at half the nuclear saturation density $\rho_{\text {tr }}=1.35 \times 10^{14} \mathrm{~g} / \mathrm{cm}^{3}$, for details see Ref. [68]. This EoS allows the larger maximum mass $M_{\mathrm{NS}}^{\max }=2.57 M_{\odot}$. For the typical NS with $M \simeq 1.4 M_{\odot}$ both of these EoS imply a radius of about $12 \mathrm{~km}$.

Although we shall restrict to these two EoSs, there is a large number of possible EoS (for reviews see e.g. Refs. [78, 79]). For instance, the phenomenological EoS suggested in Ref. [80] is stiffer, predicting the last stable configuration with $M=2.6 M_{\odot}$, while the radii of typical NS can be as large as $15 \mathrm{~km}$. From a very fundamental point of view, without appealing to a particular EoS but only requiring the micro-stability and causality conditions $0<d p / d \rho<1$, one can put an absolute upper limit on the NS mass, $M_{\mathrm{NS}}^{\max } \lesssim$ 3.2 $M_{\odot}$, known as Rhoades-Ruffini bound [81]. ${ }^{2}$

The baryon number density $n=n_{\mathrm{O}}$ is directly related to the energy density. The relation between the two densities, $n=n(\rho)$, depends on the chosen EoS. (For relevant densities the ratio $n / \rho$ is roughly constant but not exactly.) Hence, the baryon density profile can be presented as $n(r)=n[\rho(r)]$ and, integrating over the NS volume, we get the total baryon number:

$N_{\mathrm{NS}}\left(\rho_{c}\right)=4 \pi \int_{0}^{\infty} g_{r r}(r)^{1 / 2} n\left[\rho_{c} f(r)\right] r^{2} d r$

For fixed $\rho_{c}$, the shapes of $f(r)$ and $g_{r r}(r)$ depend on the EoS. The total baryon number in the NS scales with its mass nearly linearly:

$N_{\mathrm{NS}}=\kappa\left(M_{\mathrm{NS}} / m_{n}\right)=\kappa N_{\odot}\left(M_{\mathrm{NS}} / M_{\odot}\right)$

where $N_{\odot}=M_{\odot} / m_{n}=1.19 \times 10^{57}$ is the amount of baryons in the sun. E.g. the Sly EoS gives $\kappa \approx 1.1$ for the typical NS masses $M_{\mathrm{NS}} \simeq 1.4 M_{\odot}$, which increases to $\kappa \approx 1.2$ for heavy NS with masses approaching $2 M_{\odot}$. The equivalent baryonic mass is $M_{B}=m_{n} N_{\mathrm{NS}}=\kappa M_{\mathrm{NS}}$, and the mass deficit $M_{B}-M_{\mathrm{NS}}=(\kappa-1) M_{\mathrm{NS}}$ corresponds to the gravitational binding energy.

Considering now the NS gradual conversion into a MS with $N_{\mathrm{M}}<N_{\mathrm{O}}$, we find that $\rho_{\mathrm{O}}(r)>\rho_{\mathrm{M}}(r)$ at any radius and at any time, and so $M_{\mathrm{O}}>M_{\mathrm{M}}$ and $R_{\mathrm{O}}>R_{\mathrm{M}}$. Therefore, the radius of the mixed star coincides with the radius of the ordinary component, $R_{\mathrm{MS}}=R_{\mathrm{O}}$. For this reason, when solving the differential equations in Eq. (16), one has to take into account that at $r=R_{\mathrm{M}} \leq R_{\mathrm{O}}$ the pressure of $\mathrm{M}$ component vanishes meaning that its mass is saturated, i.e. $m_{\mathrm{M}}\left(r>R_{\mathrm{M}}\right)=M_{\mathrm{M}}$. Thus, at radius $R_{\mathrm{M}}$ one has to

\footnotetext{
${ }^{2}$ Non of the hadronic EoS in Refs. [78,79] can describe the NS mass $2.74 M_{\odot}$ attributed to the pulsar J1748-2021B [76]. However, the compact objects of the heavy category can be the quark stars: the EoS of quark matter could afford the masses up to $(2.5 \div 3) M_{\odot}$ or so. Nevertheless, we first restrict our considerations to the NS and discuss the role of quark stars later.
}

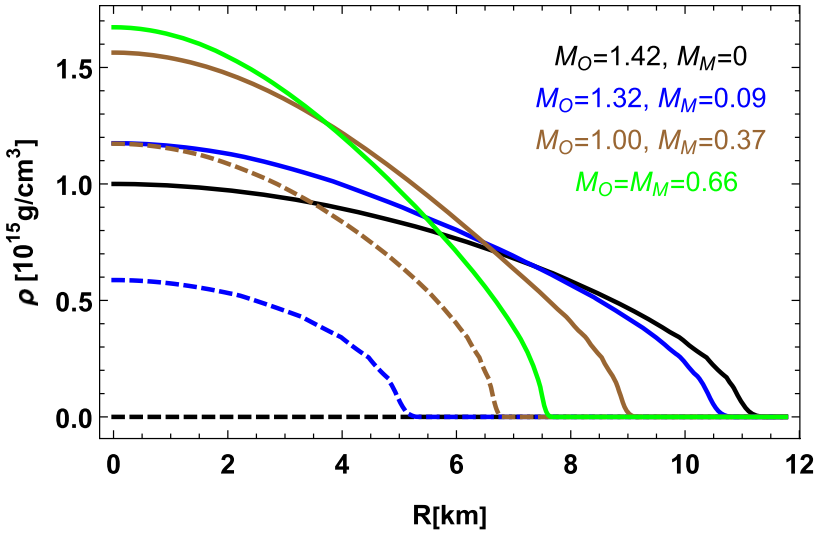

Fig. 1 Density profiles $\rho_{\mathrm{O}}(r)$ (solid) and $\rho_{\mathrm{M}}(r)$ (dashed) in a star with the initial mass $M_{\mathrm{O}} \simeq 1.42 M_{\odot}$ at different stages of its evolution from $\chi=0$ (black) to $\chi=1$ (green) with intermediate values $\chi=0.5$ and 0.75 (respectively, blue and brown). The corresponding total masses of the ordinary and mirror spheres, respectively $M_{\mathrm{O}}$ and $M_{\mathrm{M}}$, are given in the legend (in solar mass units $M_{\odot}$ ). Computations are performed using the Sly EoS [77]

stop the iteration for the differential equation of M component while continuing the integration for the $\mathrm{O}$ component up to the radius $R_{\mathrm{O}}$. The MMS configuration can be reached only asymptotically in time (provided that the star is enough light to remain stable up to this configuration, see below). In this case the $\mathrm{O}$ and $\mathrm{M}$ density profiles should be identical by symmetry, $\rho_{\mathrm{O}}(r)=\rho_{\mathrm{M}}(r)$. So, for the MMS radius and mass we should have respectively $R_{\mathrm{MMS}}=R_{\mathrm{O}}=R_{\mathrm{M}}$ and $M_{\mathrm{MMS}}=2 M_{\mathrm{O}}=2 M_{\mathrm{M}}$.

It is useful to characterize the MS configurations by the ratio of $\mathrm{M}$ and $\mathrm{O}$ central densities, $\chi=\rho_{\mathrm{cM}} / \rho_{\mathrm{cO} O}$. As far as the evolution is adiabatic and the total baryon number is conserved, for a star with a total baryon number $N=$ $N_{\mathrm{O}}+N_{\mathrm{M}}$ the value of $\chi=\chi(X)$ is determined by the mirror baryon fraction $X=N_{\mathrm{M}} / N$ at a given stage of its evolution. So an initial NS has $\chi=0$ at $X=0$, then $X$ and $\chi(X)$ increase as the star evolves, and can approach $\chi=1$ (at $X=1 / 2$ ) in the final MMS configuration.

In Fig. 1, as an example, we show the $\mathrm{O}$ and $\mathrm{M}$ density profiles obtained using the SLy EoS at different stages of the evolution at fixed baryon number $\left(N=N_{\mathrm{O}}+N_{\mathrm{M}}=1.88 \times\right.$ $\left.10^{57}\right)$, starting from the NS $(\chi=0)$ with the initial mass $M_{\mathrm{NS}}=M_{\mathrm{O}} \approx 1.42 M_{\odot}$ and radius $R_{\mathrm{NS}}=R_{\mathrm{O}} \approx 12 \mathrm{~km}$. With increasing mirror fraction the gravitational mass and the radius both gradually decrease, and when the star becomes a MMS $(X=1 / 2, \chi=1)$ its gravitational mass reduces to $M_{\mathrm{MMS}} \simeq 1.32 M_{\odot}$ while the radius shrinks to $R_{\mathrm{MMS}}=$ $R_{\mathrm{O}, \mathrm{M}} \approx 8.5 \mathrm{~km}$. Hence, in this evolution the star binding energy has increased by about $0.1 M_{\odot}$, and its radius has decreased by about $4 \mathrm{~km}$, with the increase of compactness by $30 \%$ or so. 

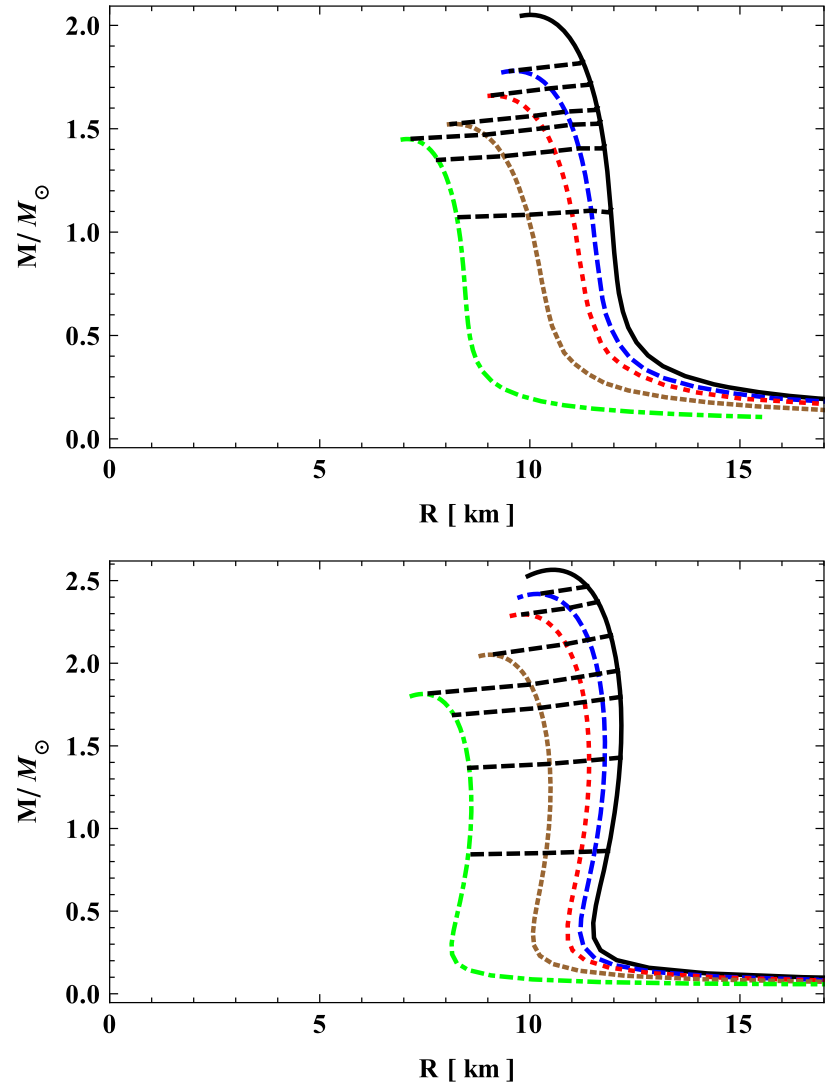

Fig. 2 Mass-radius diagrams obtained with two different EoS: Sly (upper) and joined polytropes (lower). Black solid curves correspond to initial configurations $(\chi=0)$ whereas blue, red, brown and green dashed curves correspond to mixed configurations respectively for $\chi=$ $0.5,0.6,0.75$ and 1 . Horizontal dashed curves indicate the evolution of a given NS with a conserved overall baryon number $N_{\mathrm{O}}+N_{\mathrm{M}}=N_{0}$

Very massive neutron stars cannot evolve into the MMS, since they collapse to $\mathrm{BH}$ when the ratio $\chi=\rho_{\mathrm{cM}} / \rho_{\mathrm{cO}}$ increases to some critical value. This is illustrated in Fig. 2, where we show the mass-radius relations for a mixed star with different $\mathrm{O}$ and $\mathrm{M}$ fractions (from $X=0$, solid black lines, to $X=1 / 2$, dashed green lines) for the two considered EoS. The nearly horizontal black dashed curves show the star evolution tracks with increasing $\chi$ at fixed total baryon number $N_{\mathrm{O}}+N_{\mathrm{M}}=N_{0}$. Hence, they trace the time evolution starting from the original NS $(\chi=0)$, passing the MS stage with intermediate $\chi$ 's and eventually reaching the MMS stage $(\chi=1)$. Along the evolution tracks the gravitational masses and the radii decrease making the star more compact. At a certain stage of their evolution very massive stars become gravitationally unstable forcing the collapse to $\mathrm{BH}$. The corresponding value of $\chi$ depends on the NS initial mass and on the EoS. For example, in the Sly case only the NS with initial mass $M_{\mathrm{NS}}<1.55 M_{\odot}$ or so can survive asymptotically in time (and approach the MMS configuration with $M_{\mathrm{MMS}}<1.45 M_{\odot}$ ), while the more massive stars are doomed to become BHs. E.g. a NS of initial mass $M=1.8 M_{\odot}$ collapses at $\chi \approx 0.5$.

The following remark is in order. In the above analysis we assumed that all considered objects are the NS described by the nuclear EoS. However, one cannot exclude the possibility that some fraction of the (non-BH) compact objects is represented by hypothetical hybrid stars with the quark matter core, or quark stars entirely consisting of quark matter (for a review, see e.g. [82]).

According to Bodmer-Witten hypothesis [83,84], at extreme densities the energetically favored ground state can be the strange quark matter. In this way, the NS consisting of nuclear matter can be metastable and can be transformed into the quark stars (QS) composed of deconfined quark matter. The mean lifetime of the NS is related to the nucleation time $\tau_{Q}$ to form the critical size quark-matter bubble in its central regions. Nucleation time very steeply depends on the central baryonic density $n_{\mathrm{cO}}$ and, at some threshold density $n_{\text {th }}$, it can sharply drop to values much smaller than typical pulsar ages [85-87]. In particular, considering a pure NS in which $n_{\mathrm{cO}}$ increases (e.g. due to matter fall-back after supernova explosion or due to mass accretion from the companion) and approaches $n_{\text {th }}$, a critical bubble of quark matter can be effectively formed and the star can be promptly transformed into a QS. Such NS $\rightarrow$ QS transformation can also originate the gamma-ray burst shortly after the supernova explosion $[85,86]$.

This picture implies that the heavier compact stars, in particular the ones with $2 M_{\odot}$ masses, are not the NS but they are the QS. The quark matter EoS can be stiff enough to afford the QS with rather large masses: a very general parametrization of this EoS [88] can allow the QS with masses up to $M=3 M_{\odot}$ or so, for reasonable values of two relevant parameters, the bag constant $B$ and the color-flavor locking superconducting gap $\Delta$ (the respective parameter space is shown in Fig. 1 of Ref. [89]).

The deconfinement critical density $n_{\text {th }}$ is a crucial parameter which depends on the nuclear EoS, and it determines the upper threshold $M_{\mathrm{NS}}^{\text {th }}$ for the possible NS masses. A neutron star with $M>M_{\mathrm{NS}}^{\text {th }}$ will effectively transform into a quarks star with the same baryon number, instead of collapsing to $\mathrm{BH}$. (Dynamics of NS $\rightarrow$ QS transition was studied in details in Refs. $[90,91]$.) As the NS mass approaches $M_{\mathrm{NS}}^{\text {th }}$, it promptly transforms into the QS with smaller gravitational mass, $M_{\mathrm{OS}}^{\text {th }}<M_{\mathrm{NS}}^{\text {th }}$. This is the minimal QS mass which can further increase by accretion. Therefore, in this picture (coined as two-family scenario in Ref. [92]) two types of compact stars can exist represented by the NS and QS. The NS mass distribution is limited by threshold value $M_{\mathrm{NS}}^{\text {th }}$ while the stars with $M>M_{\mathrm{NS}}^{\text {th }}$ can be only the QS. In particular, a part of the population of the recycled pulsars which accreted enough mass from the companion, or slow pulsars which suffered substantial matter fall-back after the super- 
nova explosion, can in fact be the QS. Interestingly, the stars with intermediate masses $M_{\mathrm{QS}}^{\text {th }}<M<M_{\mathrm{NS}}^{\text {th }}$ can be the NS or QS, depending on their evolution history, and only the stars with $M<M_{\mathrm{QS}}^{\text {th }}$ can be represented exclusively by the NS family.

The exact value of $n_{\text {th }}$ (and respective values of $M_{\mathrm{NS}}^{\text {th }}$ and $M_{\mathrm{QS}}^{\mathrm{th}}$ ) needs a precise calculation of the nucleation time $\tau_{Q}$ in the context of the given EoS. For example, the nuclear EoS (composed of nucleons and hyperons) considered in Refs. [93,94], without considering the possible transition to QS, would allow the maximum NS mass $M_{\mathrm{NS}}^{\max } \simeq 2 M_{\odot}$. However, taking into account the latter possibility, the threshold mass was estimated in Ref. [89] as $M_{\mathrm{NS}}^{\text {th }} \simeq 1.6 M_{\odot}$, by adopting a simple criterium that $n_{\text {th }}$ corresponds to a density at which the hyperon fraction reaches 10 per cent. Analogously, with the same criterium, the softer nuclear EoS of Ref. [95], which includes nucleons, hyperons and Delta-resonances, gives the lower value $M_{\mathrm{NS}}^{\text {th }} \simeq 1.5 M_{\odot}$. Interestingly, in both cases the corresponding baryon mass turns out to be of about $1.7 M_{\odot}$ and the QS with $M_{\mathrm{QS}}^{\text {th }} \simeq 1.3 M_{\odot}$ is produced while the transition density, depending on the model, corresponds to $n_{\text {th }} \simeq(0.5-1) \mathrm{fm}^{-3}$ [89]. Complete calculation of $n_{\text {th }}$ in the context of possible realistic EoS could give perhaps somewhat larger values of $M_{\mathrm{NS}}^{\mathrm{th}}$ and $M_{\mathrm{QS}}^{\mathrm{th}}$.

Remarkably, $n-n^{\prime}$ conversion in the QS should be ineffective, not only because of the absence of the neutrons in the quark matter (multi-quark processes as $u d d \rightarrow n^{\prime}$ could work instead), but principally because the quark matter is self-bound which makes this transition energetically inconvenient. Therefore, in the two family scenario the stars with $M>M_{\mathrm{NS}}^{\text {th }}$ can be represented exclusively by the QS family and should not manifest the effects of $n-n^{\prime}$ transition. In particular, $M-R$ evolution tracks shown in Fig. 2 will not be applicable for the (quark) stars with the initial masses larger than say $M_{\mathrm{NS}}^{\text {th }}=1.6 M_{\odot}$ or so. (Incidentally, the shape of $M-R$ diagram for the NS corresponding to the Sly EoS (upper plot in Fig. 2) is rather similar to the shape predicted by the nuclear EoS of Refs. [93,94].) In fact, the mass-radius relation of the stars above this threshold should be described by the quark matter EoS and have drastically different shape (see Fig. 1 in [89]).

However, $n-n^{\prime}$ transitions can have interesting consequences also for the NS with the birth mass below $M_{\mathrm{NS}}^{\text {th }}$. Namely, with the production of mirror neutrons in its interior, the NS shrinks and the central density $n_{\mathrm{cO}}$ of (ordinary) baryons increases. E.g. during the evolution of a NS with initial mass $M=1.42 M_{\odot}$ (see Fig. 1) the central density increases from $\rho_{\mathrm{cO}}=10^{15} \mathrm{~g} / \mathrm{cm}^{3}$ (at $\chi=0$ ) to to $\rho_{\mathrm{cO}} \approx 1.5 \times 10^{15} \mathrm{~g} / \mathrm{cm}^{3}$ (at $\chi=0.75$ ) which in the context of the Sly EoS corresponds to $n_{\mathrm{cO}} \approx 0.75 \mathrm{fm}^{-3}$. Thus, in heavier stars, after producing a significant amount of mirror matter, $n_{\mathrm{cO}}$ can reach the threshold value say $n_{\mathrm{th}}=1 \mathrm{fm}^{-3}$, and the ordinary nuclear component can be promptly trans- formed into quark matter. As for mirror component, it will remain in the hadronic form. In this way one could produce the mixed quark stars with mirror nuclear matter cores in their interior. The identification of the NS mass range in which this phenomenon can take place requires a consistent numerical calculation of the threshold density in the context of realistic EoS which will be addressed elsewhere. In the following we shall merely consider this as a possibility which, however, should have no impact for $n-n^{\prime}$ conversion for the NS with $M<M_{\mathrm{NS}}^{\text {th }}$ if the effective conversion time is much larger than the Universe age.

\subsection{Scaling relations between the neutron stars and maximally mixed stars}

The masses and radii of mixed stars depend on the central densities of the two components, $\rho_{\mathrm{cO}}$ and $\rho_{\mathrm{cM}}$. In fact, any point in the mass-radius diagram is determined by the functions $M_{\mathrm{MS}}=M\left(\rho_{\mathrm{cO}}, \rho_{\mathrm{cM}}\right)$ and $R_{\mathrm{MS}}=R\left(\rho_{\mathrm{cO}}, \rho_{\mathrm{cM}}\right)$. The dependence on two parameters causes the mass/radius degeneracy in Fig. 2, meaning that stars with the same mass can have different radii, depending on the composition.

The masses and radii along the dashed lines in Figs. 2, describing the MS evolution at fixed total baryon number $N=N_{\mathrm{O}}+N_{\mathrm{M}}$, are not related by a simple rule. However, one can find simple scaling relations between the two extreme configurations, meaning that the mass-radius trajectories corresponding to the MMS configurations (dashed green curves in Fig. 2) can be mapped onto those of the NS (black solid curves) by factor of $\sqrt{2}$ rescaling of masses and radii. In other words, the radii, masses and (total) baryon numbers of stars with $\rho_{\mathrm{cO}}=\rho_{\mathrm{cM}}=\rho_{c}$ and stars with $\rho_{\mathrm{cO}}=\rho_{c}, \rho_{\mathrm{cM}}=0$ are related as

$$
\frac{R\left(\rho_{c}, \rho_{c}\right)}{R\left(\rho_{c}, 0\right)}=\frac{M\left(\rho_{c}, \rho_{c}\right)}{M\left(\rho_{c}, 0\right)}=\frac{N\left(\rho_{c}, \rho_{c}\right)}{N\left(\rho_{c}, 0\right)}=\frac{1}{\sqrt{2}}
$$

The MS configurations can be equivalently parametrized in terms of total central density $\rho_{c}=\rho_{\mathrm{cO}}+\rho_{\mathrm{cM}}$ and the ratio $\chi=\rho_{\mathrm{cM}} / \rho_{\mathrm{cO}}$, both depending on the mirror fraction $X$. Since the NS and MMS configurations correspond to respectively $\chi=0(X=0)$ and $\chi=1(X=1 / 2)$, we can consider their radii as functions of total central density, denoting $R\left(\rho_{c}, \rho_{c}\right) \equiv R_{\mathrm{MMS}}\left(2 \rho_{c}\right)$ and $R\left(\rho_{c}, 0\right) \equiv R_{\mathrm{NS}}\left(\rho_{c}\right)$, and similarly for the masses and baryon numbers. Thus, in configurations related by $\sqrt{2}$ scaling (23) the central energy density and pressure of the MMS are twice the corresponding values of the NS.

The scaling relations are precise and they can be derived directly from the TOV equations. Since MMS contains two fluids with the identical EoS and with equal central densities, we can set $\rho_{\mathrm{O}}=\rho_{\mathrm{M}}, p_{\mathrm{O}}=p_{\mathrm{M}}$ and $m_{\mathrm{O}}=m_{\mathrm{M}}$ in Eqs. (14) and (16). Thus, both components satisfy identical differential 
equations:

$\frac{d m_{\alpha}}{d r}=4 \pi \rho_{\alpha} r^{2}, \quad \frac{1}{\rho_{\alpha}+p_{\alpha}} \frac{d p_{\alpha}}{d r}=\frac{2 m_{\alpha}+8 \pi p_{\alpha} r^{3}}{4 m_{\alpha} r-r^{2}}$

where $\alpha=\mathrm{O}$ or M. By substituting

$r=\tilde{r} / \sqrt{2}, \quad m_{\alpha}=\tilde{m}_{\alpha} / \sqrt{8}$

these equations can be rewritten as

$\frac{d \tilde{m}_{\alpha}}{d \tilde{r}}=4 \pi \rho_{\alpha} \tilde{r}^{2}, \quad \frac{1}{\rho_{\alpha}+p_{\alpha}} \frac{d p_{\alpha}}{d \tilde{r}}=\frac{\tilde{m}_{\alpha}+4 \pi p_{\alpha} \tilde{r}^{3}}{2 \tilde{m}_{\alpha} \tilde{r}-\tilde{r}^{2}}$

which have exactly the same form as the one fluid TOV equations (17) for the ordinary NS. Therefore, Eqs. (17) and (26) should have identical solutions under the same boundary conditions. Namely, taking the central densities as $\rho_{\mathrm{O}}(0)=\rho_{\mathrm{M}}(0)=\rho_{c}$, we obtain

$\rho_{\mathrm{O}}(\tilde{r})=\rho_{\mathrm{M}}(\tilde{r})=\rho_{c} f(\tilde{r})$

with exactly the same shape function as $f(r)$ in Eq. (18) but with the argument rescaled as $\tilde{r}=\sqrt{2} r$. The MMS radius corresponds to the distance at which $f(\sqrt{2} r)$ vanishes. Thus, we obtain that the radii of the MMS with central density $\rho_{\mathrm{cO}}+\rho_{\mathrm{cM}}=2 \rho_{c}$ and of the NS with the central density $\rho_{\mathrm{cO}}=\rho_{c}$ are related as

$R_{\mathrm{MMS}}\left(2 \rho_{c}\right)=\frac{1}{\sqrt{2}} R_{\mathrm{NS}}\left(\rho_{c}\right)$

On the other hand, by integrating the first equation (26) and taking into account the redefinitions (27) we get $\tilde{m}_{\alpha}(\tilde{r})=$ $\sqrt{8} m_{\alpha}(\tilde{r})=4 \pi \int_{0}^{\tilde{r}} \rho_{\alpha}(r) r^{2} d r$. Thus, for the MMS mass we have

$$
\begin{aligned}
M_{\mathrm{MMS}}\left(2 \rho_{c}\right) & =\frac{4 \pi}{\sqrt{8}} \int_{0}^{\infty}\left[\rho_{\mathrm{O}}(r)+\rho_{\mathrm{M}}(r)\right] r^{2} d r \\
& =\frac{4 \pi \rho_{c}}{\sqrt{2}} \int_{0}^{\infty} f(r) r^{2} d r=\frac{1}{\sqrt{2}} M_{\mathrm{NS}}\left(\rho_{c}\right)
\end{aligned}
$$

where the last equality follows from (19). In addition, Eqs. (28) and (29) show that the two configurations must have the same compactness:

$\frac{M_{\mathrm{MMS}}\left(2 \rho_{c}\right)}{R_{\mathrm{MMS}}\left(2 \rho_{c}\right)}=\frac{M_{\mathrm{NS}}\left(\rho_{c}\right)}{R_{\mathrm{NS}}\left(\rho_{c}\right)}$

Analogously, for the moments of inertia one can obtain:

$I_{\mathrm{MMS}}\left(2 \rho_{c}\right)=\frac{1}{2 \sqrt{2}} I_{\mathrm{NS}}\left(\rho_{c}\right)$

The following remark is required for avoiding the confusion. The NS with $\rho_{\mathrm{cO}}=\rho_{c}$ and the MMS with $\rho_{\mathrm{cO}}=$ $\rho_{\mathrm{cM}}=\rho_{c}$ are not on the same evolutionary track with the conserved baryon number (dash lines in Fig. 2). In fact, the baryon numbers of these configurations also obey to the scaling law:

$N_{\mathrm{MMS}}\left(2 \rho_{c}\right)=\frac{1}{\sqrt{2}} N_{\mathrm{NS}}\left(\rho_{c}\right)$

which can be obtained in the analogous manner, by comparing the NS baryon number (21) with the total baryon number $N_{\mathrm{O}}+N_{\mathrm{M}}=2 N_{\mathrm{O}}$ of the MMS, and taking into account that upon redefinitions (25) we have $2 m_{\alpha} / r=\tilde{m}_{\alpha} / \tilde{r}$ in $g_{r r}$ component of the metric tensor (10). By this reason also the volumes of two configurations rescale as $V_{\mathrm{MMS}} / V_{\mathrm{NS}}=$ $\left(R_{\mathrm{MMS}} / R_{\mathrm{NS}}\right)^{3}=(1 / \sqrt{2})^{3}$, as it would occur in the case of flat space.

An important implication is the following. If for given EoS the last stable NS configuration corresponds to maximum central density $\rho_{c}^{\max }, M_{\mathrm{NS}}^{\max }=M_{\mathrm{NS}}\left(\rho_{c}^{\max }\right)$, then, for the same EoS, the last stable MMS configuration would correspond to $M_{\mathrm{MMS}}^{\max }=M_{\mathrm{MMS}}\left(2 \rho_{c}^{\max }\right)$. Hence, the maximum masses (and respective baryon numbers) of the MMS and NS are related as

$M_{\mathrm{MMS}}^{\max }=\frac{1}{\sqrt{2}} M_{\mathrm{NS}}^{\max }, \quad N_{\mathrm{MMS}}^{\max }=\frac{1}{\sqrt{2}} N_{\mathrm{NS}}^{\max }$

For example, for the Sly EoS we have $M_{\mathrm{NS}}^{\max } \approx 2.05 M_{\odot}$ and $N_{\mathrm{NS}}^{\max } \approx 2.91 \times 10^{57}$, and so maximum mass and total baryon number of the MMS must be $M_{\mathrm{MMS}}^{\max } \approx 1.45 M_{\odot}$ and $N_{\mathrm{MMS}}^{\max } \approx 2.06 \times 10^{57}$. Since total baryon number is conserved in the evolution, the MMS configuration can be evolved only from the NS containing at most $N_{\mathrm{NS}} \approx$ $2.06 \times 10^{57}$ baryons, i.e. having initial mass at most $M_{\mathrm{NS}} \approx$ $1.55 M_{\odot}$ (see corresponding evolution tracks with conserved baryon numbers shown by dash lines in Fig. 2). Stars with larger initial masses collapse to $\mathrm{BH}$ at a moment of their evolution when the ratio of central densities reaches a critical value. The Rhoades-Ruffini bound $M_{\mathrm{NS}}^{\max }<3.2 M_{\odot}$ implies $M_{\mathrm{MMS}}^{\max }=M_{\mathrm{NS}}^{\max } / \sqrt{2}<2.3 M_{\odot}$.

The above scaling relations can be straightforwardly extended to scenarios with more than one mirror sector: if ordinary neutrons have mixings with $k$ "mirror" neutrons $n_{1}^{\prime}, n_{2}^{\prime}, \ldots n_{k}^{\prime}$, and if all mirror components have the same EoS as the ordinary one (E.g. such a scenario with $k \sim 10^{32}$ mirror sectors was discussed in Ref. [96]), then the NS and MMS configurations can be related by analogous $\sqrt{k}$-scaling rules. In particular, we would have $M_{\mathrm{MMS}}^{\max }=M_{\mathrm{NS}}^{\max } / \sqrt{k}$.

Let us remark that the issues discussed in this section, and in particular the scaling relations, are not directly applicable in the case of two-family scenario where the heavy stars are represented by quark stars in which $n-n^{\prime}$ transition is absent. But they remain valid for the lower mass stars in which the central density of ordinary baryons $n_{\mathrm{cO}}$ will always remain below the NS $\rightarrow$ QS transition density $n_{\text {th }}$ during the evolution to the MMS configuration. 


\section{The time evolution of mixed stars}

\section{$3.1 n-n^{\prime}$ oscillation in nuclear medium}

Let us discuss $n-n^{\prime}$ transition in the nuclear matter of the neutron stars. Their liquid core is dominantly composed of the degenerate neutrons while the protons constitute of about $10 \%$ fraction. The outer part (crust) of the NS consists mainly of the heavy nuclei, but contains also some fraction of the neutrons. Thin rigid surface layer of the crust (outer crust) is entirely built of nuclei and thus $n-n^{\prime}$ transition should be ineffective there. We assume $n-n^{\prime}$ conversion time to be much larger than the NS cooling time, so that at any stage the temperature is much less than the chemical potential.

The oscillation $n-n^{\prime}$ in a medium is described by the Schrödinger equation with effective Hamiltonian

$H=\left(\begin{array}{cc}E(p) & \varepsilon \\ \varepsilon & E^{\prime}(p)\end{array}\right)$

where the off-diagonal term $\varepsilon$ comes from $n-n^{\prime}$ mass mixing (2), and $E(p)$ and $E^{\prime}(p)$ stand for energies of $n$ and $n^{\prime}$ with the momentum $p$ (in this section we use natural units $c=1$ and $\hbar=1)$. The free-particle dispersion relation $E=\left(m_{n}^{2}+\right.$ $\left.p^{2}\right)^{1 / 2}$ is no more applicable since it is affected by the neutron coherent interactions with the matter. Therefore, the energy splitting $\Delta E=E^{\prime}-E$ is generally non-vanishing in the medium.

The Hamiltonian eigenstates are

$n_{1}=c n-s n^{\prime}, \quad n_{2}=s n+c n^{\prime}$

where $c=\cos \theta$ and $s=\sin \theta$, with $\theta$ being the mixing angle: $\tan 2 \theta=2 \varepsilon / \Delta E$. The probability of $n-n^{\prime}$ oscillation for free flight time $t$ reads $P_{n n^{\prime}}(t)=\sin ^{2} 2 \theta \sin ^{2}(\Delta E t / 2)$.

In the limit $\Delta E \rightarrow 0$ we have $\sin ^{2} 2 \theta=1$ (maximal mixing). But since the medium effects induce large energy splitting, $\Delta E \gg \varepsilon$, the mixing angle becomes small, $\theta \approx$ $\varepsilon / \Delta E \ll 1$, and we get

$P_{n n^{\prime}}(t) \approx \frac{4 \varepsilon^{2}}{\Delta E^{2}} \sin ^{2}\left(\frac{t \Delta E}{2}\right)$

For short flight times, fulfilling the condition $t<\Delta E^{-1}$, this probability becomes $\Delta E$-independent: $P_{n n^{\prime}} \approx(\varepsilon t)^{2}$. In the opposite case $t \gg \Delta E^{-1}$ oscillations can be averaged in time, and the mean oscillation probability is $P_{n n^{\prime}}=$ $\frac{1}{2} \sin ^{2} 2 \theta \approx 2 \theta^{2} \approx 2(\varepsilon / \Delta E)^{2}$. $^{3}$

\footnotetext{
3 This can be interpreted in a simple way. Creating the neutron as initial state means to create the eigenstates $n_{1}$ and $n_{2}$ respectively with the probabilities $c^{2}$ and $s^{2}$. The eigenstates do not oscillate among each other, and after freely propagating to some distance they can be detected as $n$ correspondingly with probabilities $c^{2}$ and $s^{2}$. Combining these probabilities, we get $P_{n n}=c^{4}+s^{4}$ and $P_{n n^{\prime}}=1-P_{n n}=2 c^{2} s^{2}=$ $\frac{1}{2} \sin ^{2} 2 \theta$.
}

Thus, we have to evaluate the values $t$ and $\Delta E$. We consider the NS interior as cold nuclear matter dominantly consisting of the degenerate neutrons. In the case of ideal Fermi gas we would have $E(p)=\left(m_{n}^{2}+p^{2}\right)^{1 / 2}$. However, this approximation is not sufficient for describing the observed NS (such "ideal" EoS would give the maximum NS mass $\left.M_{\mathrm{NS}}^{\max }=0.7 M_{\odot}[72]\right)$, and one has to take into account the (repulsive) nuclear interactions which stiffen the EoS of the medium. In the case of strongly interacting particles, one can discuss only the energy levels corresponding to the quantum stationary states of the Fermi liquid in whole instead of stationary states of individual particles. In other words, a particle state with given momentum has no precise meaning and one considers only the elementary excitations of the ground state $(T \rightarrow 0)$ described as quasiparticles. This makes difficult the interpretation of $n-n^{\prime}$ oscillations in the Fermi liquid. However, one can still consider a non-ideal Fermi gas switching on the nuclear interactions, provided that their 'Yukawa' radius $r_{0} \simeq 1 \mathrm{fm}$ is smaller than the mean distance between the nucleons, $n_{\mathrm{O}}^{-1 / 3} \simeq \xi^{-1 / 3} \times 2 \mathrm{fm}$, where $\xi=n_{\mathrm{O}} / n_{\mathrm{S}}$ is the baryon number density $n_{\mathrm{O}}$ in units of nuclear saturation density $n_{\mathrm{S}} \approx 0.16 \mathrm{fm}^{-3} \approx(100 \mathrm{MeV})^{3}$. In the NS interior $\xi$ can be as large as $\xi \simeq 7$ or so, and the condition $r_{0}<n_{\mathrm{O}}^{-1 / 3}$ can be only marginally satisfied. It can be also written as $p_{F} r_{0}<3$ for the Fermi momentum $p_{F}=\left(3 \pi^{2} X_{n} n_{\mathrm{O}}\right)^{1 / 3} \approx \xi^{1 / 3} \times 1.5 \mathrm{fm}^{-1} X_{n} \approx 1$ being the neutron fraction.

Strong interactions of the neutron with the target particles (mediated by single or multi-particle exchanges of the scalar and vector mesons) can be described by the effective couplings $(\bar{n} \lambda n)(\bar{N} \lambda N)$ where $\lambda=1, \gamma^{5}, \gamma^{\mu}$ etc. stand for the possible Lorentz structures and $N=n, p$ ( $N$ can be also heavier baryons at supra-nuclear densities in the NS central regions, or heavy nuclei in the NS inner crust). The coherent scatterings with the medium components modify the neutron dispersion relation as $E(p)=\left[p^{2}+\left(m_{n}-\phi\right)^{2}\right]^{1 / 2}+A_{0}$, which means that it moves in the environment of the external scalar $\phi$ and vector $A_{\mu}$ fields determined by the mean densities of target particles: $\phi \propto\langle\bar{N} N\rangle$ and $A_{0} \propto\left\langle\bar{N} \gamma^{0} N\right\rangle$. (In non-polarized matter one can set $\left\langle\bar{N} \gamma^{5} N\right\rangle=0$.) In strong coupling regime the precise values $\phi$ and $A_{0}$ are difficult to calculate and they can be estimated only by extrapolating the phenomenological potentials describing the nuclei. However, at relevant densities these values are smaller than neutron mass, and the neutrons are non-relativistic $\left(p<p_{F}=\xi^{1 / 3} \times\right.$ $\left.330 \mathrm{MeV}<m_{n}\right)$. Then, considering e.g. only the repulsive contributions, one can take $E(p)=m_{n}+\phi+A_{0}+p^{2} /\left(2 m_{n}\right)$. In other words, we take $E=m_{n}+V+p^{2} /\left(2 m_{n}\right)$ where $V$ can be accounted as in-medium optical potential

$V=2 \pi \sum_{N} a_{N} X_{N}\left(1+\delta_{n N}\right) \frac{n_{\mathrm{O}}}{m_{n}}$ 
where $a_{N}$ is the neutron scattering length on the constituent $N$ which is present with the fraction $X_{N}$ in this medium, and $\delta_{n N}$ counts for the identical particle scattering when $N=n$. In the following, for the sake of simplicity, we take $X_{n}=1$ neglecting the subdominant fraction of the protons and other components. Thus, considering only $n n$ scatterings, we have

$V=4 \pi a_{n} \frac{n_{\mathrm{O}}}{m_{n}} \approx \xi a_{n} \times 80 \mathrm{MeV} / \mathrm{fm}$

If mirror matter is also present, $n^{\prime}$ state will have analogous in-medium potential $V^{\prime}$ obtained from Eq. (38) by substituting $n_{\mathrm{O}} \rightarrow n_{\mathrm{M}}$. Hence, taking energy splitting $\Delta E=$ $V-V^{\prime}=(1-\beta) V$, where $\beta=n_{\mathrm{M}} / n_{\mathrm{O}}$, we get

$\theta=\frac{\varepsilon}{\Delta E} \approx \frac{\varepsilon m_{n}}{4 \pi a_{n} n_{\mathrm{O}}(1-\beta)}$

For simplicity, we consider exact degeneracy $m_{n^{\prime}}=m_{n}$. However our results are applicable also when $\Delta E$ is contributed by small mass splitting $m_{n^{\prime}}-m_{n} \ll V-V^{\prime}$. We also neglect the Zeeman energy induced by the neutron magnetic moment $\mu_{n}$ since $\left|\mu_{n} B\right|<10 \mathrm{keV}$ even in magnetars having magnetic fields as large as $B \sim 10^{15} \mathrm{G}$.

The neutron collisions destroy the coherence of $n-n^{\prime}$ oscillation. Therefore, the rate of $n-n^{\prime}$ transition can be estimated as $\Gamma_{n n^{\prime}}=\Gamma_{n} P_{n n^{\prime}}\left(t_{n}\right)$ where $\Gamma_{n}=1 / t_{n}$ is the neutron collision rate with the matter constituents, $t_{n}$ being the mean time between the collisions. This means that for every neutron collision the mirror neutron is produced with the probability $P_{n n^{\prime}}\left(t_{n}\right)$. Then in $t_{n} \Delta E<1$ regime, we would have $P_{n n^{\prime}}=\left(\varepsilon t_{n}\right)^{2}$ and $\Gamma_{n n^{\prime}}=\varepsilon^{2} t_{n}$. In Ref. [65] the collision time $t_{n}$ in the neutron core was considered as the time needed to travel the distance between neighbouring nucleons, $n_{\mathrm{O}}^{-1 / 3}=\xi^{-1 / 3} \times 2 \mathrm{fm}$, with a velocity $v \simeq p_{F} / m_{n} \approx \xi^{1 / 3} / 3$. In this way, without considering the effects of the Pauli blocking, one gets

$\Gamma_{n n^{\prime}}=\varepsilon^{2} t_{n}=\varepsilon_{15}^{2} \xi^{-2 / 3} \times 1.3 \cdot 10^{-15}$ year $^{-1}$

where we denote $\varepsilon=\varepsilon_{15} \times 10^{-15} \mathrm{eV}$. Since $\xi$ is position dependent (in the neutron core it can vary from 0.5 to 10 , depending on the nuclear EoS), this rate should be averaged over the NS density profile. Namely, in Ref. [65] $t_{n} \simeq 10^{-23} \mathrm{~s}$ was taken, by considering $n_{\mathrm{O}} \simeq 1 \mathrm{fm}^{-3}$ and $v / c \simeq 1 / 3$ as typical values over the star, and the mirror neutron production rate was estimated as

$\Gamma=\left\langle\Gamma_{n n^{\prime}}\right\rangle_{\mathrm{NS}} \simeq \varepsilon_{15}^{2} \times 0.6 \cdot 10^{-15} \mathrm{year}^{-1}$

However, if the condition $t_{n} \Delta E<1$ is not applicable, one has to proceed in other way, and discuss $n-n^{\prime}$ process in terms of the Hamiltonian eigenstates (35).

Although the ordinary and mirror nucleons have separate strong interactions, the mixed interactions emerge in the basis of the Hamiltonian eigenstates $n_{1}$ and $n_{2}$. E.g. the couplings $\pi^{0} \bar{n} \gamma^{5} n+\pi^{0 \prime} \overline{n^{\prime}} \gamma^{5} n^{\prime}$ with ordinary and mirror neutral pions (give rise to non-diagonal terms

$s\left(\pi^{0} \overline{n_{2}} \gamma^{5} n_{1}+\pi^{0 \prime} \overline{n_{2}} \gamma^{5} n_{1}\right)+$ h.c.

More generically, the neutron interactions with a target nucleon $(N=n, p)$ described by effective couplings $(\bar{n} \lambda n)(\bar{N} \lambda N)$ (with $\lambda=1, \gamma^{5}, \gamma^{\mu}$ etc. as possible Lorentz structures) in the eigenstate basis become

$\left(c^{2} \overline{n_{1}} \lambda n_{1}+s^{2} \overline{n_{2}} \lambda n_{2}+c s \overline{n_{2}} \lambda n_{1}+c s \overline{n_{1}} \lambda n_{2}\right)(\bar{N} \lambda N)$

which contain mixed entries between $n_{1}$ and $n_{2}$.

The mirror neutron production rate can be estimated as follows. The stationary neutron state in the medium can be viewed as the eigenstate $n_{1}$. The processes $n_{1} N \rightarrow n_{1} N$, $N=n, p$ are Pauli blocked, but $n_{1} N \rightarrow n_{2} N$ are not. Therefore, every $n_{1} n_{1}$ collision can produce $n_{2} \approx n^{\prime}$ with a cross-section $2 \theta^{2} \sigma_{n n}$ where $\sigma_{n n}=8 \pi a_{n}^{2}$ is $n n$ scattering cross-section. ${ }^{4}$ Taking the mean relative velocity as $v \simeq p_{F} / m_{n}=\left(3 \pi^{2} n_{\mathrm{O}}\right)^{1 / 3} m_{n}^{-1}$, the rate of $n \rightarrow n^{\prime}$ transition can be estimated as

$$
\Gamma_{n n^{\prime}}(\xi, \beta) \approx 2 \eta \theta^{2} \times 8 \pi a_{n}^{2} v n_{\mathrm{O}} \approx \frac{\varepsilon^{2} m_{n} \eta(\beta)}{\left(\xi n_{\mathrm{S}}\right)^{2 / 3}(1-\beta)^{2}}
$$

where for $\theta$ we used Eq. (39). Hence, the dependence on the poorly known scattering length $a_{n}$ cancels out in Eq. (44). The Pauli blocking factor $\eta(\beta)$ takes into account that the final state $n_{1}$ cannot have a momentum below the Fermi momentum $p_{F}$ whereas the momentum of the produced $n_{2} \approx n^{\prime}$ state should be above $p_{F}^{\prime}=\beta^{1 / 3} p_{F}$. The blocking factor as a function of $E_{F}^{\prime} / E_{F}=\beta^{2 / 3}$, where $E_{F}=p_{F}^{2} /\left(2 m_{n}\right)$ is the neutron "Fermi" energy, was estimated by a Montecarlo simulation of hard sphere scatterings, and the resulting dependence is reported in Fig. 3. This function has a maximum $\eta(0) \approx 0.18$ at $E_{F}^{\prime}=0$, then it decreases and, as expected, asymptotically vanishes when $E_{F}^{\prime} \rightarrow E_{F} .^{5}$

For the younger MS with $n_{\mathrm{M}} \ll n_{\mathrm{O}}$ we can set $\beta=0$ and Eq. (44) gives

$\Gamma_{n n^{\prime}}(\xi, 0) \simeq \varepsilon_{15}^{2} \xi^{-2 / 3} \times 0.7 \cdot 10^{-15} \mathrm{year}^{-1}$

This rate depends on the baryon density, meaning that $n-$ $n^{\prime}$ conversion should proceed somewhat faster in peripheral $(r \simeq R)$ low density regions of the star rather than in central (small $r$ ) region where $\xi>1$.

Mirror neutrons can be produced also by other processes. In normal conditions the mixed interaction terms (42) cannot induce decays $n_{1} \rightarrow n_{2} \pi\left(\pi^{\prime}\right)$ with the emission of neutral (ordinary and mirror) pions, simply by kinematical rea-

\footnotetext{
${ }^{4}$ The process $n_{1} n_{1} \rightarrow n_{2} n_{2}$ is not Pauli suppressed but its rate is proportional to $\theta^{4}$ and thus it is negligible. The processes $n_{1} p \rightarrow n_{2} p$ contribute with the rate $\propto \theta^{2}$, but for simplicity we neglect them because of small proton fraction in the NS interior.

${ }^{5}$ Let us remark that the reverse process $n_{2} n_{2} \rightarrow n_{2} n_{1}$ is Pauli blocked as far as $E_{F}>E_{F}^{\prime}$. This justifies why we have neglected the inverse reaction rate $\Gamma^{\prime}$ in Eq. (5) to obtain Eq. (6).
} 


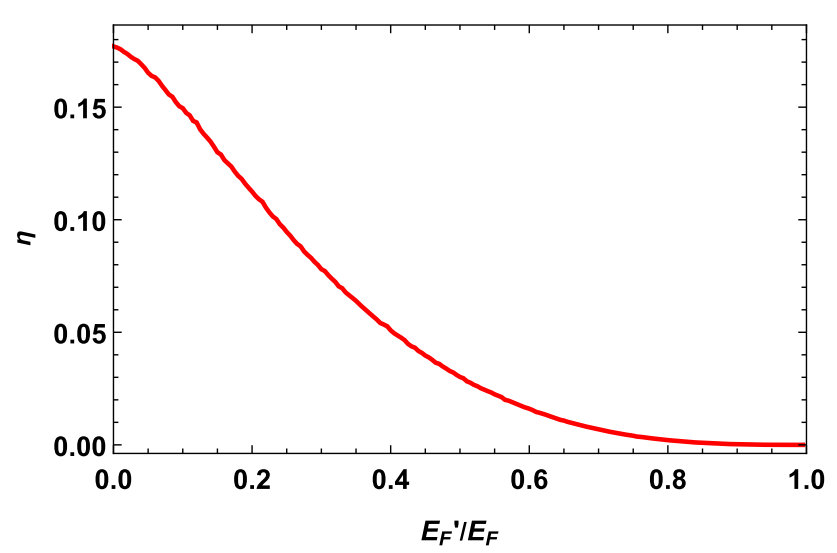

Fig. 3 Pauli blocking factor $\eta$ as a function of $E_{F}^{\prime} / E_{F}$

son. However, such decays become possible in dense nuclear medium. (This effect is similar to the matter induced neutrino decay $[97,98]$.) Namely, at large enough densities, at which the threshold condition $V>m_{\pi}$ can be satisfied, the matterinduced decays $n_{1} \rightarrow n_{2} \pi\left(\pi^{\prime}\right)$ and $n_{1} \rightarrow p^{\prime} \pi^{\prime}$, can proceed without Pauli blocking. The decay rate (again for $\beta=0$ ) is

$\Gamma_{n \rightarrow n^{\prime} \pi}=\frac{\theta^{2} g_{\pi N}^{2}\left(V-m_{\pi}\right)^{3}}{8 \pi m_{n}^{2}}=\frac{\varepsilon^{2} g_{A}^{2} V}{8 \pi F_{\pi}^{2}}\left(1-\frac{m_{\pi}}{V}\right)^{3}$

where the Goldberger-Treiman relation $g_{\pi N}=g_{A} m_{n} / F_{\pi}$ is used for the pion-nucleon coupling constant, with $F_{\pi}=$ $93 \mathrm{MeV}$ being the pion decay constant and $g_{A}=1.27$ the axial coupling constant. However, the relevant densities might not be available in the typical NS (moreover, if heavy stars are represented by the QS), and in any case the rate (46) appears to be smaller than (45).

The heavy eigenstate can also decay into the lighter one with emission of the ordinary or mirror photons, $n_{1} \rightarrow$ $n_{2} \gamma\left(\gamma^{\prime}\right)$, via the transitional magnetic moment (TMM) $\mu_{12}$ between the eigenstates [99]. The decay rates read

$\Gamma_{n \rightarrow n^{\prime} \gamma}=\Gamma_{n \rightarrow n^{\prime} \gamma^{\prime}}=\frac{\mu_{12}^{2}}{\pi} V^{3}$

In particular, the mass mixing (2) induces the TMM $\mu_{12}=$ $\theta \mu_{n}$ [64] between $n_{1}$ and $n_{2}$ states, from the magnetic moments of $n$ and $n^{\prime}, \mu_{n}=\mu_{n}^{\prime}=-1.91 \mu_{N}$, where $\mu_{N}=e / 2 m_{N}$ is the nuclear magneton. Then we get

$\Gamma_{n \rightarrow n^{\prime} \gamma^{\prime}(\gamma)}=\frac{\varepsilon^{2} \mu_{n}^{2}}{\pi} V=\varepsilon_{15}^{2} a_{n} \xi \times 10^{-51} \mathrm{GeV} / \mathrm{fm}$

which is by orders of magnitude smaller than (45). Hence, $n \rightarrow n^{\prime} \gamma$ decay cannot be the dominant effect if the TMM is induced solely by $n-n^{\prime}$ mass mixing (2). However, the situation changes if there is a direct TMM $\mu_{n n^{\prime}}$ between ordinary and mirror neutrons, in which case we would have $\mu_{12}=\mu_{n n^{\prime}}$. In fact, while the TMM between the neutron and antineutron is excluded by fundamental symmetry reasons $[100,101]$, it is allowed between $n$ and $n^{\prime}$, and it can be induced in some models of $n-n^{\prime}$ mixing [64]. Present experimental limits imply the upper bound $\mu_{n n^{\prime}}<10^{-5} \mu_{n}$ or so [99]. Clearly, for the large enough TMM, $\mu_{n n^{\prime}} \gg \theta \mu_{n}$, the rate (47) can be much larger than (45), but we do not discuss this case here.

The role of weak interactions is negligible. The Lagrangian describing mirror neutron $\beta$-decay $n^{\prime} \rightarrow p^{\prime} e^{\prime} \bar{v}_{e}^{\prime}$, induces $n_{1} \rightarrow p^{\prime} e^{\prime} \bar{v}_{e}^{\prime}$ decay via the $n_{1}$ admixture in $n^{\prime}$. It produces mirror protons and electrons without Pauli blocking but with the rate $\theta^{2} \Gamma(n \rightarrow p e \bar{v})$ which is vanishingly small as compared to (45).

Therefore, in the following our estimations will be based on the rate given by Eq. (45). (Accidentally, it agrees with a naive estimation (40) used in Ref. [65] within the factor of 2.) In any case, in view of several uncertainties in description of $n-n^{\prime}$ oscillation dense nuclear matter, this can be considered only as an order of magnitude estimation.

Clearly, all these considerations can be directly applied also to the mirror neutron stars which can evolve in mixed stars via the reverse process $n^{\prime} \rightarrow n$ producing the ordinary matter in their interior. Hence, if the conversion time is less than the age of the Universe, the old enough neutron stars, both ordinary and mirror, would end up as the MMS.

The following remark is in order. In the above we discussed $n-n^{\prime}$ transition in the NS dominated by the dense neutron matter. However, as we discussed in previous section, one cannot exclude the possibility that the heavier compact objects, and in particular the ones with masses close to $2 M_{\odot}$, are represented by quark stars for which $n-n^{\prime}$ transition is suppressed. In the case of hybrid stars, $n-n^{\prime}$ transitions can take place in the neutron liquid shell with the neutron density dependent rate (45).

\subsection{Estimating the evolution time}

Let us consider the evolution track of a star with a given overall baryon number $N_{0}=N_{\mathrm{O}}+N_{\mathrm{M}}$, starting from its initial (NS) configuration with $N_{\mathrm{O}}=N_{0}$ and $N_{\mathrm{M}}=0$. Since $n-n^{\prime}$ transition rate (44) depends on $\xi$ and $\beta$, these values should be averaged over the density distributions in the star. As far as the adiabatic evolution is assumed, at any time $t$ the density profiles $n_{\mathrm{O}}(r)=\xi(r) n_{\mathrm{S}}$ and $n_{\mathrm{M}}(r)=\beta(r) \xi(r) n_{\mathrm{S}}$, as the functions of radial coordinate $r$, and for a given star they are fully determined by the mirror fraction $X(t)=N_{\mathrm{M}}(t) / N$. Hence, the rate (44) should be averaged over the star volume, and we have $\left\langle\Gamma_{n n^{\prime}}(\xi, \beta)\right\rangle_{X}=\Gamma(X)=\Gamma_{0} \mathcal{F}(X)$ (c.f. Eq. (7)), where the 'starting' conversion rate

$\Gamma_{0}=\tau_{\varepsilon}^{-1} \approx \varepsilon_{15}^{2}\langle\xi\rangle_{X=0}^{-2 / 3} \times 5 \cdot 10^{-23} \mathrm{~s}^{-1}$

is obtained by averaging Eq. (45) over the density distribution in the initial NS ( $X=0$, i.e. $\beta=0$ ), while $X$-dependent 
factor is

$\mathcal{F}(X)=\frac{\langle\xi\rangle_{X=0}^{2 / 3}}{\eta(\beta=0)}\left\langle\frac{\eta(\beta)}{\xi^{2 / 3}(1-\beta)^{2}}\right\rangle_{X}$

where the parentheses mean the averaging over the profiles of $\xi$ and $\beta$ in the star at given mirror fraction $X$.

For a given EoS, the mass $M$ and radius $R$ of a star is determined by its conserved baryon number $N_{0}=N_{\mathrm{O}}+$ $N_{\mathrm{M}}$. For the initial NS, neglecting the GR corrections for its volume, we have $\left\langle n_{\mathrm{O}}\right\rangle_{X=0}=\langle\xi\rangle_{X=0} n_{\mathrm{S}}=N_{0} /\left(4 \pi R^{3} / 3\right)$, and we get $\langle\xi\rangle_{X=0} \simeq\left(N_{0} / N_{\odot}\right) R_{12}^{-3}$, where $R_{12}=R / 12 \mathrm{~km}$ and $N_{\odot}=1.19 \times 10^{57}$. Then from Eq. (49) we get

$$
\begin{aligned}
\tau_{\varepsilon}=\Gamma_{0}^{-1} & \simeq \varepsilon_{15}^{-2} R_{12}^{-2}\left(N_{0} / N_{\odot}\right)^{2 / 3} \times 1.5 \cdot 10^{15} \text { year } \\
& \simeq \varepsilon_{15}^{-2} R_{12}^{-2}\left(M / 1.4 M_{\odot}\right)^{2 / 3} \times 2 \cdot 10^{15} \text { year }
\end{aligned}
$$

where for the last step we used Eq. (22) taking $\kappa \approx 1.1$ for the typical NS masses $(1.2-1.6) M_{\odot}$. Using other EoS, estimation (51) can vary within factor of few.

The time at which the mirror baryon fraction in MS reaches a value $X$ is given by Eq. (8). For $X \ll 1$ the dependence is linear, and we have $t=X \tau_{\varepsilon}$ (9). E.g. for $\tau_{\varepsilon} \simeq 100 \mathrm{Gyr}$, the pulsar of a typical age $t \sim 1 \mathrm{Gyr}$ would accumulate the mirror fraction $X \sim 0.01$ equivalent to baryon mass $\sim 10^{-2} M_{\odot}$. But with growing $X$ the evolution gradually slows down since $\mathcal{F}(X)$ decreases due to increase of $\xi$ and respective decrease of Pauli factor $\eta(\beta)$.

The baryon number $N_{0}$ of the initial NS is limited by the maximal value $N_{\mathrm{NS}}^{\max }$ which depend on the EoS. (Namely, in the case of Sly EoS one has $N_{\mathrm{NS}}^{\max }=2.9 N_{\odot}$ which corresponds to $M_{\mathrm{NS}}^{\max }=2.05 M_{\odot}$.) During the evolution the central densities $\rho_{\mathrm{cM}}$ and $\rho_{\mathrm{cO}}$ both increase, as well as their ratio $\chi=\rho_{\mathrm{cM}} / \rho_{\mathrm{cO}}$, along with the $\mathrm{M}$ fraction $X$. Therefore, the star shrinks and its gravitational mass $M(X)=M_{\mathrm{O}}(X)+M_{\mathrm{O}}(X)$ decreases with $X$ growing: see Fig. 1 for a NS with initial mass $\left.1.4 M_{\odot}\right)$. However, the total baryon number is conserved during the evolution, $N_{\mathrm{O}}+N_{\mathrm{M}}=N_{0}$.

Asymptotically in time the star can approach the MMS stage with $X=1 / 2$ (i.e. $N_{\mathrm{M}}=N_{\mathrm{O}}=N_{0} / 2$ ), but this depends on its baryon number. The scaling relations of Eqs. (33) imply that only the stars with $N_{0}<N_{\mathrm{NS}}^{\max } / \sqrt{2}$ can reach the MMS configuration, having the final masses limited as $M(X=1 / 2)<M_{\mathrm{NS}}^{\max } / \sqrt{2}$. A star with $N_{0}>N_{\mathrm{NS}}^{\max } / \sqrt{2}$ should collapse to $\mathrm{BH}$ at a time $t_{\text {coll }}=t\left(X_{\mathrm{coll}}\right)$ when the mirror fraction reaches a critical value $X_{\text {coll }}$ corresponding to critical $\chi$ above which the MS canon exist (see on Fig. 2).

Figure 4 shows the time evolution of the mirror fraction $X$ in a star in units of the characteristic time $\tau_{\varepsilon}$, also highlighting the maximal possible mass that a MS can have at the age $t$. (At the moment we assume that there are no transitions into quark star.) The curves on Fig. 4, corresponding to our two
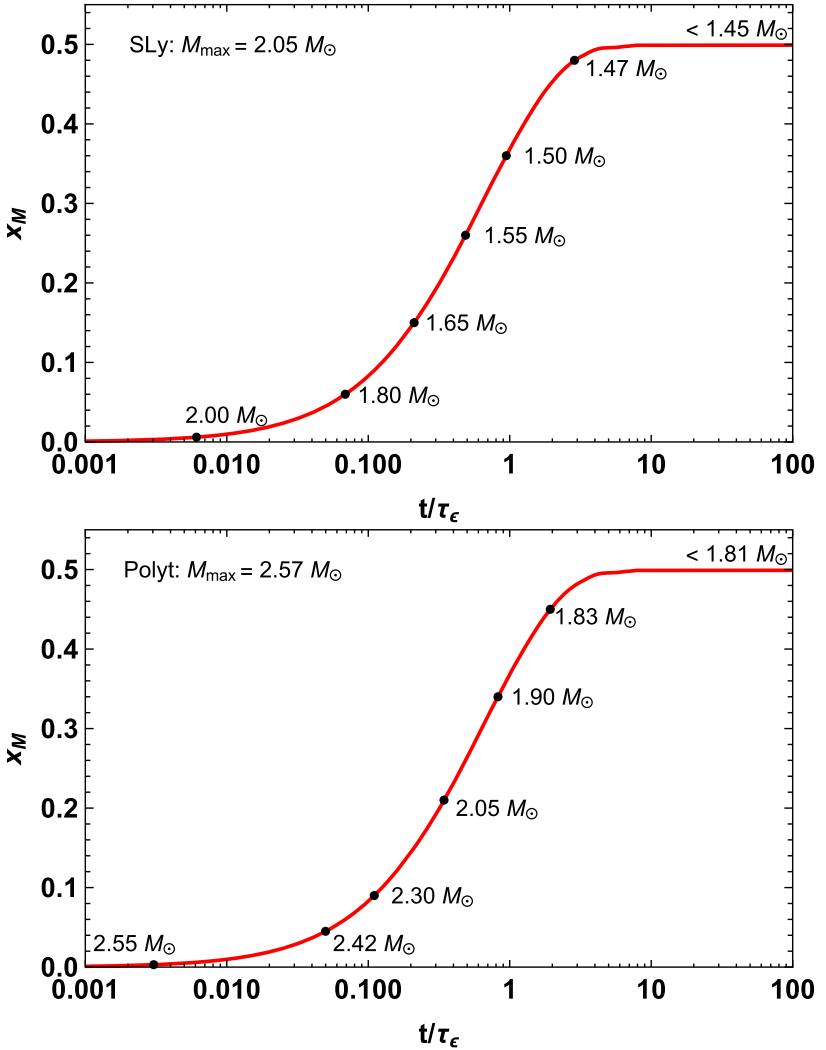

Fig. 4 Evolution of the mirror fraction $X=N_{\mathrm{M}} / N$ in mixed stars as a function of time in units of $\tau_{\varepsilon}=\Gamma_{0}^{-1}$. The black points on the solid curves show the maximal possible mass of the MS at the corresponding age

examples of EoS, Sly and joined polytrope, are obtained by numerical calculations of factors $\mathcal{F}(X)$ (50) by averaging over the density profiles of mixed stars containing a fraction $X$ of mirror baryons. For example, in the case of the Sly EoS the NS with initial mass $M \approx 1.85 M_{\odot}$ will collapse at $t \simeq 0.1 \tau_{\varepsilon}$ when its mirror fraction rises to $X \approx 0.1(\chi \approx 0.5)$ and at this moment its gravitational mass is reduced to $1.7 M_{\odot}$ or so. The stars with smaller mass can have larger lifetimes: e.g. a NS born with $M=1.65 M_{\odot}$ can survive for time $t \simeq \tau_{\varepsilon}$, and will collapse with mass reduced to $1.5 M_{\odot}$. As for the stars with initial masses $M \leq 1.55 M_{\odot}$ they do not collapse during the evolution, and asymptotically in time they can reach the MMS configuration with masses $M(X=$ $1 / 2)<M_{\mathrm{NS}}^{\max } / \sqrt{2} \approx 1.45 M_{\odot}$, gaining the gravitational binding energy of about $0.1 M_{\odot}$.

The evolution shown in Fig. 4 assumes that all compact objects, irrespectively of their masses, are the NS dominated by hadronic matter. However, as was already mentioned, the heavy stars, and in particular the ones of $2 M_{\odot}$ masses can be quark stars in which $n-n^{\prime}$ transition is ineffective. Namely, in the two-family (NS + QS) scenario $n-n^{\prime}$ oscillation, by increasing the central density, can trigger the NS to QS transition (instead of NS to BH transition). Interestingly, this 
can occur (without matter accretion) also for the NS with $M<M_{\mathrm{NS}}^{\text {th }}$. The identification of the NS evolution track in this scenario requires a special study in the context of realistic EoS which will be addressed elsewhere. Obviously, no $n-n^{\prime}$ conversion can occur after NS to QS transition when ordinary component becomes a quark matter and no neutrons remain in the star. As for mirror component produced before the NS to QS transition, it can remain in the form of mirror hadronic matter. In this case ordinary and mirror components in the star are no more symmetric and they should be described by the different EoS.

Let us now estimate the energy produced in a star by $n-n^{\prime}$ transition. Since the process $n n \rightarrow n n^{\prime}$ takes place at the momenta close to the Fermi surface, the typical energy of produced $n^{\prime}$ should be about the Fermi energy, $E_{n^{\prime}} \simeq E_{F}$. Therefore, the "starting" (at $X=0$ ) rate of the energy production, which is applicable if $t \ll \tau_{\varepsilon}$, can be obtained by multiplying the baryon amount in the NS, $N \approx\left(M / 1.4 M_{\odot}\right) \times 1.8 \cdot 10^{57}$, on the rate of $n-n^{\prime}$ transition $\Gamma_{0}=\tau_{\varepsilon}^{-1}$ and on the average energy $\left\langle E_{F}\right\rangle_{X=0} \simeq\langle\xi\rangle_{X=0}^{2 / 3} \times 10^{-4} \mathrm{erg}$. Taking also $\langle\xi\rangle_{X=0} \simeq\left(N / N_{\odot}\right) \simeq 1.5 \times\left(M / 1.4 M_{\odot}\right)$, we obtain 6

$\dot{\mathcal{E}}_{n n^{\prime}} \simeq\left(\frac{10^{15} \text { year }}{\tau_{\varepsilon}}\right)\left(\frac{M}{1.4 M_{\odot}}\right)^{5 / 3} \times 7 \cdot 10^{30} \mathrm{erg} / \mathrm{s}$

The produced energy should be radiated away by cooling in terms of $\mathrm{M}$ photons and neutrinos, and produced $n^{\prime}$ will form a mirror matter core. In fact, mirror neutrons produced at very initial stages will decay as $n^{\prime} \rightarrow p^{\prime} e^{\prime} \bar{v}^{\prime}$ and cooling can be due to mirror neutrino emission. At further stages the nucleosynthesis processes involving $p^{\prime}$ and $n^{\prime}$ will begin producing mirror nuclei. If by time $t$ the density of $\mathrm{M}$ core becomes sufficiently large, then it will undergo the 'neutronization' forming the liquid of mirror neutrons. In addition, with $t$ approaching $\tau_{\varepsilon}$, the energy emission rate starts to drop and it vanishes when $t \gg \tau_{\varepsilon}$, i.e. for stars that achieved final MMS stage.

Eq. (52) can be compared to the energy radiation rate due to the magnetic dipole field

$\dot{\mathcal{E}}_{\text {magn }}=\frac{1}{6} B_{\alpha}^{2} R^{6} \Omega^{4} \simeq B_{9}^{2} R_{12}^{6} P_{10}^{-4} \times 3 \cdot 10^{33} \mathrm{erg} / \mathrm{s}$

Here $\Omega=2 \pi / P$ is the pulsar rotation angular frequency ( $P_{10}=P / 10 \mathrm{~ms}$ ) and $B_{\alpha}=B \sin \alpha$, where $B$ is the magnetic

\footnotetext{
6 This is a rough estimation since the additional energy produced by the rearrangement of the density profile (shrinking) of the star is neglected; mirror neutrons produced in the star in fact should gain additional kinetic energy when they fall to the NS centre. Notice also that in this estimation we did use Eq. (49) expressing $\Gamma_{0}$ in terms of $\varepsilon$, since it was obtained in a model dependent way, with strongly assumptions on the mechanism of $n-n^{\prime}$ conversion in dense nuclear matter. (In fact, by taking $\Gamma_{n n^{\prime}}(\xi, 0) \propto$ $\xi^{-2 / 3}$ from Eq. (45), we would obtain the product $\Gamma_{n n^{\prime}}(\xi, 0) E_{F}(\xi) \simeq$ $\varepsilon_{15}^{2} \times 5 \cdot 10^{-27} \mathrm{erg} / \mathrm{s}$ to be $\xi$-independent.) In other words, we consider Eq. (52) which relates the energy production rate to the transition time $\tau_{\varepsilon}$ to be more robust than the relation (49) between $\tau_{\varepsilon}$ and $\varepsilon$.
}

field strength at the pole and $\alpha$ is the angle between the pulsar magnetic moment and its rotation axis $\left(B_{9}=B_{\alpha} / 10^{9} \mathrm{G}\right)$. If $\tau_{\varepsilon}>10^{15}$ year, then for typical pulsars one expects $\dot{\mathcal{E}}_{n n^{\prime}} \ll \dot{\mathcal{E}}_{\text {magn }}$. Let us note that $\dot{\mathcal{E}}_{n n^{\prime}}(52)$ is a rather regular quantity which depends only on the inferred value of $\tau_{\varepsilon}$, while $\dot{\mathcal{E}}_{\text {magn }}$ (53) depends on the pulsar rotation frequency and its magnetic field which can vary by orders of magnitude between different pulsars. Hence, for some pulsars the two rates can be comparable and in some cases even $\dot{\mathcal{E}}_{n n^{\prime}}>\dot{\mathcal{E}}_{\text {magn }}$ is possible.

\section{Comparison with astrophysical observations}

Let us discuss now the possible astrophysical implications of $n-n^{\prime}$ conversion in the neutron stars and derive limits on the transformation time $\tau_{\varepsilon}$ which in turn can be translated into the bounds on $\varepsilon$. Most of these effects are model dependent, and one should be careful in their interpretation. Namely, they can depend on the EoS, and for some stars they can depend on the evolution history and/or specific environmental conditions. For the sake of simplicity, we shall discuss two possible situations. First we discuss the case when the NS transformation time $\tau_{\varepsilon}$ is larger than the age of the universe $t_{U}$. In this case all observed NS should be still under evolution, being dominated by normal nuclear matter and hosting only a small mirror fraction proportional to their age $t, X=t / \tau_{\varepsilon}$. We shall discuss effects of $n-n^{\prime}$ transformation for the pulsar mass loss and heating. Then we shall concentrate on the possibility of $\tau_{\varepsilon}$ being much smaller than $t_{U}$, in which case the old stars with $t>\tau_{\varepsilon}$ should be already converted into the MMS.

\subsection{Effects of slow $n-n^{\prime}$ transformation: $\tau_{\varepsilon}>t_{U}$}

The pulsar ages can be estimated assuming that the spindown rate of the pulsar rotation is dominated by the energy radiated by a rotating dipole magnetic moment. If this is the only braking mechanism, the pulsar age $t$ is given by the relation

$t=\frac{P(t)}{2 \dot{P}(t)}\left[1-\left(\frac{P(0)}{P(t)}\right)^{2}\right]$

where $P(t)$ is the rotation period at the time $t$ and $P(0)$ is the rotation period at the star birth $t=0$. The measurable value $\tau_{c}=P /(2 \dot{P})=-\Omega /(2 \dot{\Omega})$, usually called the pulsar characteristic (or spin-down) age, coincides with the true age if the pulsar rotation period at birth was much smaller than at present, $P(0) \ll P(t)$, and if the magnetic dipole emission was always dominant over other slowing down processes. For most of known pulsars, more prominently for those which 
Table 1 Some of most massive pulsars, with their measured $P$ and $\dot{P}$ values and derived characteristic ages $\tau_{c}=P / 2 \dot{P}$

\begin{tabular}{|c|c|c|c|c|}
\hline PSR & $M\left[M_{\odot}\right]$ & $P[\mathrm{~ms}]$ & $\dot{P}\left[10^{-20}\right]$ & $\tau_{c}[\mathrm{Gyr}]$ \\
\hline $\mathrm{J} 1614-2230$ [73] & $1.97(04)$ & 3.1508 & 0.962 & 5.2 \\
\hline J0348+0432 [74] & $2.01(04)$ & 39.123 & 24.07 & 2.6 \\
\hline $\mathrm{J} 0740+6620[75]$ & $2.14(09)$ & 2.8857 & 1.219 & 3.8 \\
\hline $\mathrm{J} 1748-2021 \mathrm{~B}[76]$ & $2.74(21)$ & 16.760 & -32.91 & -1.1 \\
\hline
\end{tabular}

were observed with large spin-down rates $\dot{P} / P$, the true age $t$ can be rather close to their characteristic age $\tau_{c}$.

The heaviest 'old' pulsars observed up to now, with their characteristic ages, are listed in Table 1. The masses of first three pulsars in this Table are compatible, within the error bars, with the Sly EoS $\left(M_{\mathrm{NS}}^{\max }=2.05 M_{\odot}\right)$. Thus, for this EoS these pulsars would collapse $n-n^{\prime}$ in very short time $t<\tau_{\varepsilon} / 200$ or so, as its is shown on upper Fig. 4. while for our second EoS their lifetime can be $\sim \tau_{\varepsilon} / 2$ or so. Thus, if the true ages of these pulsars correspond to their spin-down ages $\tau_{c}$, typically of few Gyr, the very existence of these pulsars would imply that $\tau_{\varepsilon}>t_{U}=14$ Gyr.

The case of the globular cluster pulsar J1748-2021B (or NGC 6440B) is most challenging. In spite of large errors in the mass determination: $M=(2.74 \pm 0.21) M_{\odot}$ [76], already its existence excludes the SLy EoS. (However, conservatively thinking, there is $1 \%$ probability of low enough inclination in which case the pulsar mass can be below $2 M_{\odot}$ [76].) Within $1.5 \sigma$ error its mass can be $2.4 M_{\odot}$ which is compatible with our second example, the piecewise polytrope. Now, from lower Fig. 4 we see that star of this mass had to collapse within the time $t=\tau_{\varepsilon} / 20$ or so. Unfortunately, the true age of PSR J1748-2021B cannot be determined since its measured $\dot{P}$ value is negative: instead of spinning-down, this pulsar seemingly spins-up. It is also possible that this pulsar is rather young, created e.g. by the gravitational merger of two typical NS. More radically, in the context of two-family scenario, all heavy pulsars as in Table 1 should be the quark stars and thus they should be insensitive to $n-n^{\prime}$ mixing.

Let us notice that in the presence of $n-n^{\prime}$ conversion the NS true age and its characteristic age are no more related via Eq. (54). In fact, the latter equation is obtained by integrating the pulsar evolution differential equation

$\dot{\Omega}=-\frac{B_{\alpha}^{2} R^{6}}{6 I} \Omega^{3}$

which is obtained by equating the energy radiation rate (53) with the time derivative of the pulsar rotational energy $\mathcal{E}_{\text {rot }}=$ $I \Omega^{2} / 2$, and assuming that the moment of inertia $I$ remains constant in time: $\dot{\mathcal{E}}_{\text {rot }}=I \Omega \dot{\Omega}$. From this equation, using the measurable values $\Omega$ and $\dot{\Omega}$, one can also determine the pulsar magnetic field and the energy loss rate (53). Let us remark, however, that these are derived parameters obtained under assumption that the loss of rotational energy is dominated by the magnetic dipole radiation. E.g., for millisecond pulsar J1614-2230 the derived values of surface magnetic field and spin-down luminosity were evaluated respectively as $B \simeq$ $2 \times 10^{8} \mathrm{G}$ and $\dot{E}_{\mathrm{magn}} \simeq 10^{34} \mathrm{erg} / \mathrm{s}$ [73].

In the presence of $n-n^{\prime}$ conversion $I$ is not constant. Hence, $\dot{\mathcal{E}}_{\text {rot }}=I \Omega \dot{\Omega}+\dot{I} \Omega^{2} / 2$ and the pulsar evolution differential equation should be modified as

$\dot{\Omega}=-\frac{B_{\alpha}^{2} R^{6}}{6 I} \Omega^{3}-\frac{\dot{I}}{2 I} \Omega$

The value $\dot{I} / I=\dot{M} / M+2 \dot{R} / R$ is negative as far as in the process of $n-n^{\prime}$ transformation the star mass and radius both decrease. If the second term in Eq. (56) is significant, then Eq. (54) becomes invalid and the pulsar age $t$ is no more related to its spin-down time $\tau_{c}$. This suggests that some pulsars could have low spin-down rates not because they are very old and posses very small magnetic fields, but because of partial cancellation between the first (negative) and second (positive) terms in (56). In addition, the actual values of magnetic fields can be larger than the derived ones, and the true ages of these pulsars can be less than their spindown ages.

Moreover, if the positive contribution $-(\dot{I} / 2 I) \Omega$ is dominant in Eq. (56), then we would have $\dot{\Omega}>0$. In particular, it is tempting to propose that the negative $\dot{P}$ of PSR J1748-2021B in Table 1 is originated from this effect, meaning that this pulsar is spinning-up instead of slowing down (though this effect could be mimicked also by the gravitational acceleration in globular cluster). Interestingly, globular clusters NGC6440 and NGC6441 contain other pulsars with $\dot{P}<0$, one with a light companion :J1748-2021F, and two isolated ones: J1748-2021C and J1750-3703C [76]. If future observations will find the isolated spinning-up pulsars, with the intrinsic value $\dot{P} / P$ being confidently negative after subtracting the acceleration effects, then this could be interpreted as the effect of the star contraction due to $n-n^{\prime}$ transformation.

The interesting observation of Ref. [65] is that the star mass loss due to $n-n^{\prime}$ transition should gradually increase the orbital period $P_{b}$ in pulsar binaries, as

$$
\frac{\dot{P}_{b}^{\mathrm{nn}}}{P_{b}}=-\frac{2\left(\dot{M}+\dot{M}_{c}\right)}{M+M_{c}}
$$


where $M$ is the NS mass and $M_{c}$ is the mass of its companion which can be e.g. a white dwarf of another neutron star. In this way, the observational data on the orbital period decay $\dot{P}_{b} / P_{b}$ can be used to obtain the limits on the evolution time $\tau_{\varepsilon}$.

In fact, several pulsar binaries have positive measured values of $\dot{P}_{b}$. However, apart of positive contribution $\dot{P}_{b}^{n n^{\prime}}$ by $n-n^{\prime}$ transition, there are other dynamical effects to be considered. Namely, the observed value should be corrected for the contributions related to the system acceleration in the galactic gravitational potential, kinematic Shklovskii effect of apparent acceleration and, for compact systems, also for the quadrupolar emission of gravitational waves $(\mathrm{GW})$ : $\dot{P}_{b}=\dot{P}_{b}^{n n^{\prime}}+\dot{P}_{b}^{\text {gal }}+\dot{P}_{b}^{\text {kin }}+\dot{P}_{b}^{\mathrm{GW}}$. For example, binary system J0437-4715, containing one of the most stable pulsars with $M \approx 1.4 M_{\odot}$ and its light companion with $M_{c} \approx 0.25 M_{\odot}$, has $P_{b}=5.741$ days and $\dot{P}_{b}^{\text {obs }}=(3.73 \pm 0.06) \times 10^{-12}$ [102]. Hence, after subtracting the above effects, and taking into account that the $\mathrm{GW}$ emission is negligible for this system, one obtains

$\frac{\dot{P}_{b}^{n n^{\prime}}}{P_{b}}=\frac{\dot{P}_{b}-\dot{P}_{b}^{\text {gal }}-\dot{P}_{b}^{\text {kin }}}{P_{b}}=(1.0 \pm 1.8) \times 10^{-11} /$ year

which is compatible with vanishing effect: we have $\dot{P}_{b} / P_{b}<$ $2.8 \times 10^{-11} /$ year within $1 \sigma$ error. Assuming $\dot{M}_{c}=0$ (meaning that the companion mass loss is negligible), from Eq. (57) we obtain $|\dot{M} / M|<1.7 \times 10^{-11} /$ year.

Somewhat stronger limit can be obtained from the HulseTaylor pulsar B1913+16 known as a perfect binary system for testing General Relativity. The masses of the pulsar and its companion (presumably another NS) were determined with a great precision: $M=1.4398(2) M_{\odot}$ and $M_{c}=$ $1.3886(2) M_{\odot}$, as well as the orbital period and its derivative: $P_{b}=0.322997$ days and $\dot{P}_{b}=-2.423(1) \times 10^{-12}$ [103]. For this compact binary, the GW contribution is predicted to be large, $\dot{P}_{b}^{\mathrm{GW}}=-2.402531(14) \times 10^{-12}$, which almost saturates the observed value. After subtracting also the galactic correction $\dot{P}_{b}^{\text {gal }}=(-0.027 \pm 0.005) \times 10^{-12}$, one obtains

$$
\frac{\dot{P}_{b}^{n n^{\prime}}}{P_{b}}=\frac{\dot{P}_{b}-\dot{P}_{b}^{\mathrm{GW}}-\dot{P}_{b}^{\mathrm{gal}}}{P_{b}}=(7.4 \pm 5.6) \times 10^{-13} / \text { year }
$$

Then, assuming that both companions are the NS and, having nearly equal masses, both suffer identical mass losses, from (57) we get $\dot{M} / M \approx\left(\dot{M}+\dot{M}_{c}\right) /\left(M+M_{c}\right)=-(3.7 \pm$ $2.8) \times 10^{-13} /$ year. We interpret this result as an upper bound $|\dot{M} / M|<6.5 \times 10^{-13} /$ year.

The above limits can be translated to the lower bounds on the evolution time $\tau_{\varepsilon}$. Namely, assuming that $\tau_{\varepsilon}>t_{U}$, the mass loss rate by the NS can be estimated as

$$
\frac{\dot{M}}{M}=\frac{\dot{X}}{M} \frac{d M}{d X} \approx-0.19 \times \tau_{\varepsilon}^{-1}
$$

where we plugged $\dot{X}=1 / \tau_{\varepsilon}(9)$. The value $(d M / d X)_{X=0}$ was found by numerical calculation using the Sly EoS for a star with initial mass $1.4 M_{\odot}$. Namely, we computed the mass difference $\Delta M(X)=M(X)-M(0)$ between the mixed star with small mirror fraction $X$ and the original NS, and extrapolated the value $\Delta M(X) / X$ to $X \rightarrow 0$. Configurations of this star corresponding to larger values of $X$ are shown in Fig. 1.

In this way, the above limit on PSR B1913+16 translates into a lower bound $\tau_{\varepsilon}>300$ Gyr. Using Eq. (51), it can be translated into upper bound $\varepsilon<8 \times 10^{-14} \mathrm{eV}$ or so. PSR J0437-4715 gives a weaker limit $\tau_{\varepsilon}>13 \mathrm{Gyr}$, i.e. $\varepsilon<$ $4 \times 10^{-13} \mathrm{eV}$, which nicely coincides with the result of Ref. [65]. Since the above pulsars belong to a light category with well determined masses $M \simeq 1.4 M_{\odot}$, it is rather improbable that they can be quark stars. Therefore, the limit from the mass loss argument $\varepsilon<10^{-13}$ or so, which was obtained in Ref. [65] and which we confirm here, should be robust.

Energy production due to $n-n^{\prime}$ transition can lead to other interesting effects. As it was discussed in Sect. 3, per each $n n \rightarrow n n^{\prime}$ process the mirror neutron $n^{\prime}$ is produced with typical Fermi energy $E_{F}=\xi^{2 / 3} \times 60 \mathrm{MeV}$. For $\tau_{\varepsilon}>t_{U}$ all neutron stars can be considered as young, and the energy production rate $\dot{E}_{n n^{\prime}}$ is given by Eq. (52). This energy will be radiated away in terms of mirror photons $\gamma^{\prime}$ and neutrinos $v^{\prime}$. At first instants the mirror matter in the NS has very small density and the produced $n^{\prime}$ will preferably decay as $n^{\prime} \rightarrow p^{\prime} e^{\prime} \bar{v}^{\prime}$ producing a hot plasma of mirror protons and electrons gravitationally trapped inside the neutron star. At later stage, with increase of mirror density, various nucleosynthesis processes start to proceed in hot medium of mirror nucleons. In this way, ordinary NS should be visible for mirror observers via the mirror photon emission. Reciprocally, $n^{\prime} \rightarrow n$ conversion will produce ordinary matter in the interior of mirror NS, so that it can becomes visible for us as a source of hard photon emission, in the far UV and X-ray ranges.

On the other hand, some part of energy produced in the NS will be emitted in terms of ordinary photons and neutrinos. Namely, disappearance of the neutron in the reaction $n n \rightarrow$ $n n^{\prime}$ leaves the "empty" level in the Fermi see which will be filled by transition of the neutron from the higher level. Once again, since $n n \rightarrow n n^{\prime}$ reactions take place close to the Fermi surface, the transition energies should be by an order of magnitude smaller than the Fermi energy $E_{F}$. Thus, the ordinary component should get somewhat less heating than the mirror one, and we consider that energy emitted in in terms of ordinary photons corresponds to a fraction $x \simeq 0.1$ of total luminosity $\dot{\mathcal{E}}_{n n^{\prime}}$ given by Eq. (52). Then the 
NS surface temperature can be estimated as

$T \simeq\left(\frac{10^{15} \text { year }}{\tau_{\varepsilon}}\right)^{1 / 4}\left(\frac{M}{1.4 M_{\odot}}\right)^{5 / 12} \times 1.5 \cdot 10^{5} \mathrm{~K}$

simply by equating $x \dot{E}_{n n^{\prime}}=4 \pi R^{2} \cdot \sigma T^{4}$, with $\sigma$ being the Stefan-Boltzmann constant, and taking $R \approx 12 \mathrm{~km}$ for the NS radius.

The standard cooling mechanisms predict sharp drop of the temperature with the age of the star, implying $T \simeq 10^{4} \mathrm{~K}$ after $10^{7}$ year and $T \simeq 10^{3} \mathrm{~K}$ after $10^{8}$ year. Interestingly, observations of some old pulsars detect that they are still warm, with the surface temperatures $10^{5} \mathrm{~K}$ or larger. For example, above discussed PSR J0437-4715 is the brightest millisecond pulsar in UV and X-rays. Its characteristic age is $\tau_{c}=3.2$ Gyr but its UV spectral shape suggests a thermal emission with $T=(1.5 \div 3.5) \times 10^{5} \mathrm{~K}$ [104]. A solitary $5 \mathrm{~ms}$ pulsar PSR J2124-3358, with $\tau_{c}=3.8 \mathrm{Gyr}$, has a thermal spectrum with $T=(0.5 \div 2) \times 10^{5} \mathrm{~K}$ [105], as well as an younger pulsar B0950+08 $\left(\tau_{c}=1.8 \times 10^{7}\right.$ year $)$ with $T=(1 \div 3) \times 10^{5} \mathrm{~K}$ [106]. These temperatures are much higher than predicted by standard cooling models, which means that some heating mechanism operates in the NS, which in the context of our model can be related to $n-n^{\prime}$ transformation with $\tau_{\varepsilon} \sim 10^{15}$ year. Using Eq. (51), we obtain corresponding $n-n^{\prime}$ mass mixing as $\varepsilon \sim 10^{-15}$ $\mathrm{eV}$ or so. It should be noted, however, that the determination and interpretation of the NS surface temperatures are model dependent. They depend on interstellar extinction and the models of pulsar magnetosphere, accretion from the partner and non-thermal emission. In addition, there are also other possible heating mechanisms related e.g. to Urca processes with vortex friction and rotochemical reactions.

On the otter hand, observations of slow pulsar J2144-3933 ( $P=8.5 \mathrm{~s}$ ) with $\tau_{c}=3.3 \mathrm{Gyr}$ imply solely an upper bound $T<4.2 \times 10^{4} \mathrm{~K}$ [107] which disfavors some of heating mechanisms but still remains compatible with $n-n^{\prime}$ transformation with $\tau_{\varepsilon}>6 \cdot 10^{16}$ year or so. However, the suppressed thermal spectrum in PSR J2144-3933 can be related also to uncertain environmental factors, and it would be premature to derive any serious conclusion. Moreover, one cannot exclude the possibility that this isolated pulsar, with unknown mass, is in fact the quark star in which case $n-n^{\prime}$ transition would have no effects for it. Therefore, the limit on $\tau_{\varepsilon}$ obtained from PSR J2144-3933 on the basis of just one non-detection can be taken with a proper caution.

\subsection{Fast $n-n^{\prime}$ transformation: $\tau_{\varepsilon}<10^{5}$ year}

Let us discuss now the situation when the NS transformation time is rather small, say $\tau_{\varepsilon}<10^{5}$ year. Then the old stars with the ages $t>10^{5}$ year should be already transformed into the asymptotic MMS, with (almost) equal amounts of ordinary and mirror baryons inside. So old stars would not not manifest effects of $n-n^{\prime}$ transformation. The transition times larger than $10^{5}$ year are disfavored by the arguments discussed in previous section. Namely, the limit on the orbital period decay in the case of PSR J1141-6545, relatively young pulsar with $\tau_{c} \approx 2 \times 10^{6}$ year, excludes the transition times in the range $10^{6}<\tau_{\varepsilon}<10^{11}$ year or so. The pulsar heating is also important: according to Eq. (52), for $\tau_{\varepsilon}=10^{5}$ the energy luminosity of a pulsar with $t<\tau_{\varepsilon}$ exceeds the solar luminosity by seven orders of magnitude. However, for pulsars younger than $10^{5}$ year the cooling should be rather fast, with the produced energy radiated away dominantly by the neutrinos while the photon emission can be by orders of magnitude smaller. Hence, one cannot directly exclude the possibility of $\tau_{\varepsilon}<10^{5}$ year or so, though the determination of an exact upper limit on $\tau_{\varepsilon}$ from the pulsar heating requires a special study.

A clear phenomenological implication of the gravitational mass scaling (33) is that (for any nuclear EoS) the maximal possible mass of the MMS should be $\sqrt{2}$ times smaller then the maximum mass of pure NS. As far as old stars with $t \gg \tau_{\varepsilon}$ should be the MMS, then their masses all should be below $M_{\mathrm{MMS}}^{\max }=M_{\mathrm{NS}}^{\max } / \sqrt{2}$. For example, our first example $\left(\right.$ SLy EoS, $\left.M_{\mathrm{NS}}^{\max } \approx 2.05 M_{\odot}\right)$ implies $M_{\mathrm{MMS}}^{\max } \approx 1.45 M_{\odot}$, while for the second example (piecewise politrope) gives $M_{\mathrm{NS}}^{\max } \approx 2.57 M_{\odot}$ and respectively $M_{\mathrm{MMS}}^{\max } \approx 1.81 M_{\odot}$. Similar bounds emerge from various realistic EoS reviewed in Refs. [78,79]. Thus, non of these EoS (as well as various realistic EoS reviewed in Refs. [78,79]) can afford the existence of the MMS with $M>1.8 M_{\odot}$ or so.

On the other hand, old pulsars with masses $\geq 2 M_{\odot}$ do exist (see Table 1) which constitutes a challenge. E.g. PSR J0740+6620, with $M=(2.14 \pm 0.09) M_{\odot}$ could be MMS only in the context of very stiff EoS allowing $M_{\mathrm{NS}}^{\max } \approx$ $3 M_{\odot}$ which almost saturates the Rhoades-Ruffini bound $M_{\mathrm{NS}}^{\max }<3.2 M_{\odot}$. As for PSR J1748-2021B with $M=$ $(2.74 \pm 0.21) M_{\odot}$, its interpretation as MMS is clearly inconsistent with the Rhoades-Ruffini bound which for the MMS maximum mass implies $M_{\mathrm{MMS}}^{\max }<2.3 M_{\odot}$. However, as was already mentioned, the determination errors are large the apparent large mass of this object can be related to the uncertainties in the inclination etc. [76]. In addition, this pulsar could in fact be rather young (recall that it has a negative $\dot{P}$ and so its age remains unknown) so that can speculate that it was born in relatively recent time e.g. by gravitational merger of two typical neutron stars. Anyway, the possibility of fast NS conversion into the MMS of $2 M_{\odot}$ masses is in tension with the existing nuclear EoS, and requires unrealistically stiff one practically saturating the Rhoades-Ruffini bound.

The two family (NS+QS) scenario fully avoids this controversy since the compact stars with masses above $M_{\mathrm{NS}}^{\text {th }}$ all should be the quark stars. This picture has two advantages. First that the quark matter EoS can be stiff enough to afford 
rather heavy stars, with masses up to $(2 \div 3) M_{\odot}$. Second that in quark stars $n-n^{\prime}$ transformation should be suppressed for two reasons: first because there are not much neutrons to transform, and second because the quark matter is selfbound (as standard nuclei). Therefore their transition to mirror nuclear matter should give no energy gain, in particular if quark matter is in color superconducting phase [108,109].

However, for lighter stars the two family scenario offers interesting possibilities for $n-n^{\prime}$ transition. In this case the NS can be only the stars having the central baryon density below a (nuclear EoS dependent) threshold value, $n_{\mathrm{cO}}<$ $n_{\text {th }}$, which in turn determines the NS highest possible mass $M_{\mathrm{NS}}^{\text {th }}$ and respective amount of baryons $N_{\mathrm{NS}}^{\text {th }}$. (As already discussed, the heavier objects, with baryon number $N_{0}>$ $N_{\mathrm{NS}}^{\mathrm{th}}$, all should be the QS.) However, an initially stable NS, with $N_{0}<N_{\mathrm{NS}}^{\text {th }}$ and say $R=12 \mathrm{~km}$, during its evolution can accrete an sufficient amount of mass to increase its baryon number to $N_{\mathrm{NS}}^{\mathrm{th}}$ (and correspondingly its mass to $M_{\mathrm{NS}}^{\mathrm{th}}$ ). But at this point it would undergo the phase transition to a more tightly bound quark matter and this NS with a mass $M_{\mathrm{NS}}^{\text {th }}$ will promptly transform into the QS with much smaller mass $M_{\mathrm{QS}} \simeq 0.8 M_{\mathrm{NS}}^{\mathrm{th}}$ (but with larger radius [110]), irradiating the huge amount of energy corresponding to the mass deficit.

If the NS does not accrete the mass, $N_{0}<N_{\mathrm{NS}}^{\text {th }}$ remains conserved, and it can evolve as a MS by $n-n^{\prime}$ transitions producing mirror baryons in its interior. With the increase of mirror fraction $X$ in time, the star gradually shrinks and its central density $n_{\mathrm{cO}}$ increases. Correspondingly, as depicted in Fig. 2, the star radius decreases during the evolution process and its mass becomes somewhat smaller with respect to the initial value, due to the gain in the gravitational binding energy.

In view of scaling relation (33), in the absence of the NS $\rightarrow$ QS transition the stars with $N_{0}<N_{\mathrm{NS}}^{\max } / \sqrt{2}$ can evolve to a MMS stage $X=1 / 2$, whereas the stars with larger $N_{0}$ should collapse to $\mathrm{BH}$ at smaller $X$. However, considering the possibility of the NS $\rightarrow$ QS transition, the star, even having $N_{0}<N_{\mathrm{NS}}^{\max } / \sqrt{2}$, can approach the MMS stage only if $n_{\mathrm{cO}}$ remains smaller than $n_{\text {th }}$ for all its configurations from $X=0$ to $X=1 / 2$. E.g. for a MS evolution shown in Fig. 1 the central baryon density increases from $n_{\mathrm{Oc}}=0.55 \mathrm{fm}^{-3}$ to $n_{\mathrm{Oc}}=0.75 \mathrm{fm}^{-3}$ during its evolution from the NS $(X=0)$ to the MMS $(X=1 / 2)$. Thus, if the NS $\rightarrow$ QS transition threshold density is say $n_{\text {th }} \approx 1 \mathrm{fm}^{-3}$, this star with the initial mass $M_{\mathrm{NS}}=1.4 M_{\odot}$ and radius $R_{\mathrm{NS}}=12 \mathrm{~km}$ can evolve to an asymptotic MMS configuration with a final mass $M_{\mathrm{MMS}} \approx 1.3 M_{\odot}$ and radius $R_{\mathrm{MMS}} \approx 8.5 \mathrm{~km}$.

However, if meantime, at some time before reaching the MMS stage $X=1 / 2$, the central density $n_{\text {Oc }}$ reaches the threshold density $n_{\text {th }}$ then the MS will be transformed into a less compact mixed QS in which ordinary component will be in the form of quark matter, and the produced mirror fraction $X<1 / 2$ will remain in the form of nuclear matter. Hence, the hadronic matter transition to quark matter can be triggered by $n-n^{\prime}$ conversion even for the NS having masses somewhat below $M_{\mathrm{NS}}^{\mathrm{th}}$. E.g. for $n_{\mathrm{th}} \approx 1 \mathrm{fm}^{-3}$ this can be the case for the NS with $M_{\mathrm{NS}}>1.5 M_{\odot}$ or so.

The young stars (with $t<\tau_{\varepsilon}$ ) should still be under $n-n^{\prime}$ transformation process. Thus they must gradually shrink decreasing their radii. This can manifest in observational phenomena as e.g. pulsar "glitches", sudden increase of the rotation frequency caused by irregular transfer from the NS interior to the crust and by the post "star quake" rearrangement of the crust. In fact, $n-n^{\prime}$ conversion which proceeds adiabatically in the NS liquid inner parts is not followed by the adiabatic shrinking of the rigid crust which instead ruptures in discrete events. Depending on the situation, such effects can cause also pulsar "anti-glicthes", events of a sudden spin-down. It is tempting to think that also the phenomena of soft gamma repeaters or intermittent pulsars can be related to the effects of $n-n^{\prime}$ conversion during the evolution.

Reassuming, in the two family scenario all stars with masses $M$ larger than threshold mass, say $M_{\mathrm{NS}}^{\text {th }} \simeq 1.6 M_{\odot}$ can be only the QS, with the radii that can vary in the range $R=(13 \div 15) \mathrm{km}$ for $M$ varying from $1.6_{\odot}$ to $2.5 \odot$ or so (for concreteness, we take reference values as in Ref. [89]). The QS should be stable against $n-n^{\prime}$ process which is ineffective in quark matter. The young stars $\left(t \ll \tau_{\varepsilon}\right)$ with $M \leq 1.4 M_{\odot}$ should be the NS with $R=12 \mathrm{~km}$, while the older stars of the same mass can be the MS with radii in the range $(8.5 \div 12) \mathrm{km}$ with lower value corresponding to the MMS (if $t>\tau_{\varepsilon}$ ). However, also the mixed QS can exist dominantly consisting of ordinary quark matter and partly of mirror nuclear matter. These configurations can be obtained from $\mathrm{MS} \rightarrow \mathrm{QS}$ transformation triggered by $n-n^{\prime}$ conversion which can increase central density $n_{\mathrm{Oc}}$ to the phase transition threshold $n_{\text {th }}$. The possibility of such transformation depends on the NS birth and evolution history, and it can be the case for the NS with the initial masses in the range $(1.5 \div 1.6) M_{\odot}$ or so. Paradoxically, such mixed QS can be rather light, with masses perhaps as small as $1.1 M_{\odot}$, and with radii of about $12 \div 13 \mathrm{~km}$.

Unfortunately, for the compact stars whose masses are known with a high precision, the radii remain practically unknown. Few cases when masses and radii are both determined have very poor precision [78]. But if the precise measurements by the NICER mission, see e.g. in Ref. [111], will detect very different radii in the wide range $8 \div 13 \mathrm{~km}$ irrespectively of the objects mass, this could be interpreted as a possible manifestation of fast $n-n^{\prime}$ conversion.

The double neutron star (DNS) binaries represent an interesting case for studying the effect of the $n \rightarrow n^{\prime}$ conversion on the mass distribution of compact stars. These NS are thought to have received little or no accretion, thereby reflecting the NS stellar mass at birth with a direct link to the super- 
nova mechanism. The observed DNS masses can be nicely fitted with a gaussian with a central value at $\approx 1.35 M_{\odot}$ and rather small dispersion, $\sigma \approx 0.05 M_{\odot}$ [78], though it is not quite clear why a general supernova explosion should lead to such a central mass and to such a peaked mass distribution. In fact, the gravitational masses for collapsed cores could have much wider distribution which can be further altered by the matter fall-back or subsequent mass accretion.

The characteristic ages of stars in DNS systems are larger than $10^{8}$ year or so, and thus for $\tau_{\varepsilon}<10^{6}$ year they can be considered as the MMS stars. The effect of $n \rightarrow n^{\prime}$ conversion process is to shift the peak of the mass distribution at birth towards lower values [63]. Therefore, if one could prove that the NS mass distribution at birth is wide, extending up to values $1.6 M_{\odot}$ or more, then their narrow distribution in the DNS systems could manifest either the NS $\rightarrow$ MMS transformation or the NS $\rightarrow$ QS transition, both processes leading to smaller final masses.

In the above we discussed $n \rightarrow n^{\prime}$ transformation of ordinary NS into mixed stars. However, reciprocally via $n^{\prime} \rightarrow n$ transition also mirror neutron stars should evolve into mixed stars, with the same transformation time $\tau_{\varepsilon}$ Thus, the final MMS configuration can be obtained starting either from ordinary NS or mirror NS. As far as for $\tau_{\varepsilon}<10^{5}$ year most of the stars with $M<1.4 M_{\odot}$ should be the MMS which essentially are configurations of superimposed neutron stars, ordinary and mirror, bounded to each other by gravitation, in principle they can be 'visible' for both ordinary and mirror observers.

In first approximation, the observed old pulsars, with $\tau_{c}>\tau_{\varepsilon}$, should be those MMS which were originated from the ordinary NS possessing the ordinary magnetic field, and thus are visible via their electromagnetic radiation. However, also the mirror-born MMS could be visible for us and they can be detectable as ordinary pulsars. provided that by some mechanism they can acquire the large ordinary magnetic field.

This can naturally be induced by kinetic mixing term between ordinary and mirror photons $(\epsilon / 2) F^{\mu \nu} F_{\mu \nu}^{\prime}$ [19] which effectively makes mirror electrons and protons minicharged (with ordinary electric charges $\sim \epsilon$ ), with interesting implications for the dark matter direct detection $[112,113]$. The cosmological bounds imply $\epsilon<5 \times 10^{-9}$ or so [114] while the experimental limit from the positronium decays is yet weaker: $\epsilon<5 \times 10^{-8}$ [115]. The neutrons produced in a mirror NS via $n^{\prime} \rightarrow n$ transition at first stages of its evolution, will decay into electrons and protons. Subsequently the nuclear reactions will take place producing the nuclei but some fraction of electrons will always remain in the star. Then the mirror NS rotation, via Rutherford-like scatterings due to the photon kinetic mixing, will drag the electrons rather than protons and ions, inducing circular electric currents which can give rise to substantially large magnetic fields by the mechanism suggested in Ref. [116]. In this way, it can become a complicated task how to distinguish between the old pulsars initially originated from ordinary and mirror NS since they should also have comparable surface temperatures. One possibility is that "mirror-born" pulsars will dominantly accrete mirror matter, since their companions in binaries should be $\mathrm{M}$ stars, and so they should be active in terms of mirror X-rays rather than in ordinary X-rays. However, the pulsars in the DNS systems (as well as their companions) in principle could have ordinary or mirror origin.

\section{Neutron star mergers and associated signals}

Let us briefly discuss the implications on $n-n^{\prime}$ transitions for gravitational wave (GW) bursts from the NS mergers and the associated electromagnetic signals as gamma ray burst (GRB) and kilonova events which are also known as the main source of production of heavy (trans-iron) elements in the Universe.

LIGO Collaboration detected two candidates. The first event GW170817 [117] is considered as a clear signal of the ordinary NS merger, with masses of two stars $M_{1,2}$ compatible with $\simeq 1.4 M_{\odot}$ and their total mass $M_{\text {tot }}=$ $(2.75 \pm 0.02) M_{\odot}$ typical for the DNS binaries. Remarkably, the GW signal was accompanied also by a weak GRB as well as by electromagnetic afterglows in different diapasons.

The second candidate GW190425 [118] is rather unusual. While the best fit masses of individual components $\left(M_{1} \simeq\right.$ $1.8 M_{\odot}$ and $M_{2} \simeq 1.6 M_{\odot}$ ) are within the mass range of the observed NS, both the source-frame chirp mass (1.44 \pm $0.02) M_{\odot}$ and the total mass $(3.4 \pm 0.2) M_{\odot}$ of this system are significantly larger than those of any other known DNS binaries. In addition, no confirmed electromagnetic event has been identified in association with this GW signal which suggests that this event could be originated by the merging mirror NS [119-121].

The possibility of $n-n^{\prime}$ conversion adds new features to the picture. Namely, if the conversion time $\tau_{\varepsilon}$ is short, then the old neutron stars should exist today in the MMS form, with equal amounts of the $\mathrm{O}$ and $\mathrm{M}$ components inside. Therefore, the MMS mergers should have potentially observable electromagnetic counterparts irrespectively of their origin (ordinary or mirror). In fact, one cannot exclude the possibility that GW170817 event was induced by coalescence of the stars which were initially born as the mirror NS and later evolved into the MMS.

As for GW190425, the location of this merger is practically unknown since the GW was essentially detected only by the LIGO Livingston interferometer: the LIGO Hanford at this moment was off-line while the signal of Virgo was at the level of noise. Thus, the non-detection of the associated electromagnetic counterpart does not really exclude that it was a 
merger of the two MMS. In addition, one can also speculate on the unusual mass parameters of this system and consider that GW190425 was a coalescence of the mirror quark stars. In quark matter $n-n^{\prime}$ transition is suppressed, and coalescence of the mirror QS, with null or small amount of ordinary matter inside, should not have any potentially detectable electromagnetic association. In fact, the quark stars can be rather typically produced after the collapses of mirror stars, due to its their (helium dominated) chemical content [15] which renders their evolution and pre-collapse conditions different from those of ordinary stars [18].

The enhanced compactness of the MMS with respect to NS can have implications for the GW signals. Namely, the GW waveforms from the coalescence are sensitive to the tidal deformations that each components gravitational field induces on its companion, and thus it can give relevant information about the EoS and the radii. In particular, the analysis of GW170817 signal favors the softer EoS as e.g. Sly [77], and the limits on tidal deformability for the component masses $1.4 M_{\odot}$ implies their radii $R>10 \mathrm{~km}$ [122]. This is larger than the MMS radii $R_{\mathrm{MMS}}=8.5 \mathrm{~km}$ predicted by the scaling relation (28). However, it would be premature to make strong conclusions before a solid statistics is achieved on the GW signals. For example, this DNS system could be rather young and its components are still in evolution, so that their radii could be larger than $10 \mathrm{~km}$. Moreover, other interpretations were also discussed with different implications for the identity of this system [123].

If the transition time is larger than the age of the universe, $\tau_{\varepsilon} \gg t_{U}$, no MMS can be formed and only a small fraction of mirror matter can be produced in the neutron stars during their lifetime. Thus, all coalescing stars should have the radius $R \approx 12 \mathrm{~km}$. The mirror matter forms a small core which is bound inside the NS by gravity, without having material friction with the dominant ordinary component. Therefore, every sudden collision with external bodies or fast accretion of a large chunk of matter could cause relative vibrations between the two components, which may manifest as some sort of glitches. Therefore, when the dominant $\mathrm{O}$ components of a merging binary hit each other and coalesce, the small mirror cores can be kicked out from the merger site by a sling-like effect and, having masses smaller than the evaporation limit $0.1 M_{\odot}$ or so, they should explode due to decompression. This can give rise to the kilonova-type events which can be seen by mirror observers.

The gravitational mergers of mirror neutron stars can have different and intriguing implications in the case when $\tau_{\varepsilon} \gg t_{U}$. In the absence of $n-n^{\prime}$ mixing, the mirror NS wold contain no ordinary matter, and thus the mergers of the mirror DNS binaries would produce gravitational wave signals without being accompanied by any standard electromagnetic counterpart. In other words, these mergers would look as 'invisible but not silent'. But in our scenario $n^{\prime} \rightarrow n$ transitions will produce small ordinary cores inside the mirror NS. Therefore, when the dominant mirror components of a merging binary hit each other and coalesce, their ordinary cores will be kicked out and will explode due to decompression. This explosions give rise to a hot neutron-rich cloud around the coalescence site undergoing intense r-processes. In other words, we suggest that the observed kilonova events as well as weak gamma ray bursts, or at least some of them, could originate from the merging of the mirror neutron stars with a small fraction of ordinary nuclear matter in their interiors.

Another intriguing possibility is that mirror matter has the baryon asymmetry of the opposite sign relative to ordinary matter, so that mirror neutrons inside transform into the standard anti-neutrons rather than into the neutrons, $n^{\prime} \rightarrow \bar{n}$ [124]. Such a situation can be naturally realized in co-baryogenesis models between $\mathrm{O}$ and $\mathrm{M}$ sectors discussed in Refs. [32,125]. In this case the cores of the mirror NS should consist of ordinary antimatter, so that their gravitational mergers and decompression of their antimatter cores can be at the origin of anti-r processes produce ordinary anti-nuclei. Electromagnetic signals cannot distinguish between the kilonova and anti-kilonova events. However, the produced anti-nuclei can be hunted by the AMS2 Collaboration in the spectrum of cosmic rays.

\section{Conclusions}

We have discussed the possibility that the ordinary neutron stars, via $n-n^{\prime}$ conversion, can develop the mirror matter cores which gradually increase in time. Only the stars with masses less than some (the EoS dependent) critical value can survive asymptotically in time reaching the maximally mixed configuration: the heavier stars should collapse into black holes or quark stars.

To distinguish from other works, let us remark that the neutron stars with small mirror cores formed by the dark matter accretion was discussed in Refs. [126,127]. However, dark matter accretion cannot be very effective and it can destabilize only heaviest pulsars with masses already very close to maximum mass for the given EoS. In fact, the neutron star with a bigger rate should accrete the normal matter in which interior it was born, as it is the case for the recycled neutron stars.

The neutron star conversion into dark neutron star induced by the neutron-dark neutron mixing was discussed in Refs. $[128,129]$. The dark neutron was considered as an elementary particle, without significant self-interaction, and with mass close to the neutron mass. It was shown that the masses of such dark stars cannot be larger than $0.7 M_{\odot}$. (In fact, this result is known since the seminal work of Oppenheimer and Volkoff [72] where the maximum NS mass was determined as 
$M_{\mathrm{NS}}=0.7 M_{\odot}$, assuming a simplest (but non-realistic) EoS of the ideal Fermi-degenerate neutron gas.) For stabilizing these objects, the dark neutron self-interactions were ad hoc introduced in Ref. [130], which possibility for dark matter stars was previously studied also in Ref. [131].

In our case, once the concept of mirror matter is adopted which has the same EoS as the ordinary matter, there is no need for complementary hypotheses for guaranteeing the stability of the mixed stars. Therefore, the existence of the maximally mixed stars, with evenly distributed $\mathrm{O}$ and $\mathrm{M}$ components, implies only the upper limit on their maximum mass (33). This limit also depends on the chosen EoS, and it can be avoided if the heavy pulsars are considered as quark stars in which $n-n^{\prime}$ transition is ineffective.

We have discussed various astrophysical implications of this scenario. First we discussed the situation when the NS transformation time is larger than the universe age, $\tau_{\varepsilon}>t_{U}$, in which case all existing NS should be under the evolution processes. We have shown that the limits on the pulsar timings, orbital period changes in binary systems and the pulsar surface temperatures, yield the lower bound $\tau_{\varepsilon}>10^{15}$ year. This in turn implies an upper limit on $n-n^{\prime}$ mass mixing, $\varepsilon<10^{-15} \mathrm{eV}$ or so. For free $n-n^{\prime}$ oscillations, which is the target of several ongoing and planned experiments on the neutron disappearance $\left(n \rightarrow n^{\prime}\right)$ or regeneration $\left(n \rightarrow n^{\prime} \rightarrow n\right)$, this corresponds to characteristic times $\tau_{n n^{\prime}}=\varepsilon^{-1}>1 \mathrm{~s}$.

On the other hand, the possibility of rather fast transformation cannot be excluded, with $\tau_{\varepsilon}<10^{5}$ year or so. In this case for the NS of typical ages $10^{6} \div 10^{10}$ year are already transformed into the equilibrium configuration of the MMS and so they should no more manifest effects of $n-n^{\prime}$ transition. The younger pulsars with $t<10^{5}$ year still should be under evolution and can have a substantial heating by $n-n^{\prime}$ conversion. In this case there can exist compact stars of the same mass but with the radii varying in wide range, from $8 \mathrm{~km}$ to $12 \mathrm{~km}$ or so. This possibility can be tested by the NICER radius measurements. The transition time $\tau_{\varepsilon} \sim 10^{5}$ year corresponds $\varepsilon \sim 10^{-10} \mathrm{eV}$. In this case $n-n^{\prime}$ oscillations can be of phenomenological interest for explaining the neutron lifetime problem provided that $n$ and $n^{\prime}$ have a small mass splitting $\sim 100 \mathrm{neV}$ [60]. Several experiments are underway for testing this possibility.

The evolution times in the intermediate range $\tau_{\varepsilon}=\left(10^{5} \div\right.$ $10^{15}$ ) year are excluded, though more careful analysis is needed to more accurately determine both the lower and upper edges of the excluded area.

We have also briefly discussed the effects of $n-n^{\prime}$ conversion for quark stars, and shown that also mixed QS can exist with some admixture of mirror nuclear matter (or reciprocally mirror QS with an admixture of ordinary nuclear matter). We also discussed implications for the gravitational mergers in binary systems. Interesting possibility is that also the coa- lescence of mirror-born neutron stars could give rise to the weak GRB and associated kilonova events.

Acknowledgements We thank Alessandro Drago for many useful discussions. The work of Z.B. was supported in part by by the Ministero dell'Istruzione, Università e della Ricerca (MIUR) under the program PRIN 2017, Grant No. 2017X7X85K "The Dark Universe: A Synergic Multimessenger Approach", and in part by Shota Rustaveli National Science Foundation (SRNSF), Grant DI-18-335/New Theoretical Models for Dark Matter Exploration.

Data Availability Statement This manuscript has no associated data or the data will not be deposited. [Author's comment: This is a theoretical work. No experimental data were used.]

Open Access This article is licensed under a Creative Commons Attribution 4.0 International License, which permits use, sharing, adaptation, distribution and reproduction in any medium or format, as long as you give appropriate credit to the original author(s) and the source, provide a link to the Creative Commons licence, and indicate if changes were made. The images or other third party material in this article are included in the article's Creative Commons licence, unless indicated otherwise in a credit line to the material. If material is not included in the article's Creative Commons licence and your intended use is not permitted by statutory regulation or exceeds the permitted use, you will need to obtain permission directly from the copyright holder. To view a copy of this licence, visit http://creativecomm ons.org/licenses/by/4.0/.

Funded by $\mathrm{SCOAP}^{3}$.

\section{References}

1. T.D. Lee, C.N. Yang, Question of parity conservation in weak interactions. Phys. Rev. 104, 254 (1956)

2. I.Y. Kobzarev, L.B. Okun, I.Y. Pomeranchuk, On the possibility of experimental observation of mirror particles. Sov. J. Nucl. Phys. 3(6), 837 (1966)

3. R. Foot, H. Lew, R.R. Volkas, A model with fundamental improper space-time symmetries. Phys. Lett. B 272, 67 (1991)

4. Z. Berezhiani, Mirror world and its cosmological consequences. Int. J. Mod. Phys. A 19, 3775 (2004). [arXiv:hep-ph/0312335]

5. Z. Berezhiani, Through the looking-glass: Alice's adventures in mirror world, in From Fields to Strings, Circumnavigating Theoretical Physics, vol. 3, ed. by M. Shifman et al. (World Scientific, Singapore, 2005), pp. 2147-2195. arXiv:hep-ph/0508233

6. R. Foot, Mirror dark matter: cosmology, galaxy structure and direct detection. Int. J. Mod. Phys. A 29, 1430013 (2014). arXiv: 1401.3965

7. L.B. Okun, Mirror particles and mirror matter: 50 years of speculations and search. Phys. Usp. 50, 380 (2007). arXiv:hep-ph/0606202

8. Z. Berezhiani, A.D. Dolgov, R.N. Mohapatra, Asymmetric inflationary reheating and the nature of mirror universe. Phys. Lett. B 375, 26 (1996). arXiv:hep-ph/9511221

9. Z. Berezhiani, Astrophysical implications of the mirror world with broken mirror parity. Acta Phys. Polon. B 27, 1503 (1996). arXiv:hep-ph/9602326

10. Z. Chacko, H.S. Goh, R. Harnik, The Twin Higgs: natural electroweak breaking from mirror symmetry. Phys. Rev. Lett. 96, 231802 (2006). arXiv:hep-ph/0506256

11. S.I. Blinnikov, M.Y. Khlopov, Possible astronomical effects of mirror particles. Sov. Astron. 27, 371 (1983) 
12. M.Y. Khlopov, G.M. Beskin, N.E. Bochkarev, L.A. Pustylnik, S.A. Pustylnik, Observational physics of mirror world. Sov. Astron. 35, 21 (1991)

13. H.M. Hodges, Mirror baryons as the dark matter. Phys. Rev. D 47, 456 (1993)

14. R.N. Mohapatra, S. Nussinov, V.L. Teplitz, Mirror matter as selfinteracting dark matter. Phys. Rev. D 66, 063002 (2002). arXiv:hep-ph/0111381

15. Z. Berezhiani, D. Comelli, F. Villante, The early mirror universe: inflation, baryogenesis, nucleosynthesis and dark matter. Phys. Lett. B 503, 362 (2001). arXiv:hep-ph/0008105

16. A.Y. Ignatiev, R.R. Volkas, Mirror dark matter and large scale structure. Phys. Rev. D 68, 023518 (2003). arXiv:hep-ph/0304260

17. Z. Berezhiani, P. Ciarcelluti, D. Comelli, F. Villante, Structure formation with mirror dark matter: CMB and LSS. Int. J. Mod. Phys. D 14, 107 (2005). arXiv:astro-ph/0312605

18. Z. Berezhiani, S. Cassisi, P. Ciarcelluti, A. Pietrinferni, Evolutionary and structural properties of mirror star MACHOs. Astropart. Phys. 24, 495 (2006). arXiv:astro-ph/0507153

19. B. Holdom, Two U(1)'s and epsilon charge shifts. Phys. Lett. B 166, $196(1986)$

20. S.L. Glashow, Phys. Lett. B 167, 35-36 (1986)

21. Z. Berezhiani, Unified picture of the particle and sparticle masses in SUSY GUT. Phys. Lett. B 417, 287-296 (1998). arXiv:hep-ph/9609342

22. B. Belfatto, Z. Berezhiani, How light the lepton flavor changing gauge bosons can be. Eur. Phys. J. C 79(3), 202 (2019). arXiv: 1812.05414

23. E.K. Akhmedov, Z.G. Berezhiani, G. Senjanovic, Phys. Rev. Lett. 69, 3013 (1992). arXiv:hep-ph/9205230

24. R. Foot, H. Lew, R. Volkas, Possible consequences of parity conservation. Mod. Phys. Lett. A 7, 2567 (1992)

25. R. Foot, R. Volkas, Neutrino physics and the mirror world: how exact parity symmetry explains the solar neutrino deficit, the atmospheric neutrino anomaly and the LSND experiment. Phys. Rev. D 52, 6595 (1995). arXiv:hep-ph/9505359

26. Z.G. Berezhiani, R.N. Mohapatra, Reconciling present neutrino puzzles: sterile neutrinos as mirror neutrinos. Phys. Rev. D 52, 6607 (1995). arXiv:hep-ph/9505385

27. Z.K. Silagadze, Neutrino mass and the mirror universe. Phys. Atom. Nucl. 60, 272-275 (1997). arXiv:hep-ph/9503481

28. L. Bento, Z. Berezhiani, Leptogenesis via collisions: the lepton number leaking to the hidden sector. Phys. Rev. Lett. 87, 231304 (2001). arXiv:hep-ph/0107281

29. L. Bento, Z. Berezhiani, Baryogenesis: the lepton leaking mechanism. Proc. XI Int. School. Particles and Cosmology, Baksan Valley, Russia, 18-24 Apr., 2001. arXiv:hep-ph/0111116

30. L. Bento, Z. Berezhiani, Baryon asymmetry, dark matter and the hidden sector. Fortsch. Phys. 50, 489 (2002)

31. Z. Berezhiani, Unified picture of ordinary and dark matter genesis. Eur. Phys. J. ST 163, 271 (2008)

32. Z. Berezhiani, Matter, dark matter, and antimatter in our Universe. Int. J. Mod. Phys. A 33(31), 1844034 (2018)

33. Z. Berezhiani, L. Bento, Neutron-mirror neutron oscillations: how fast might they be? Phys. Rev. Lett. 96, 081801 (2006). arXiv:hep-ph/0507031

34. Z. Berezhiani, More about neutron-mirror neutron oscillation. Eur. Phys. J. C 64, 421 (2009). arXiv:0804.2088

35. Z. Berezhiani, Neutron-antineutron oscillation and baryonic majoron: low scale spontaneous baryon violation. Eur. Phys. J. C 76(12), 705 (2016). arXiv: 1507.05478

36. S. Fajfer, D. Susič, Colored scalar mediated nucleon decays to an invisible fermion. Phys. Rev. D 103(5), 055012 (2021). arXiv:2010.08367

37. V. Kuzmin, CP violation and baryon asymmetry of the universe. Pisma Zh. Eksp. Teor. Fiz. 12, 335 (1970)
38. R.N. Mohapatra, R.E. Marshak, Local B-L symmetry of electroweak interactions, Majorana neutrinos and neutron oscillations. Phys. Rev. Lett. 44, 1316 (1980)

39. D.G. Phillips et al., Neutron-antineutron oscillations: theoretical status and experimental prospects. Phys. Rep. 612, 1 (2016). arXiv: 1410.1100

40. K.S. Babu et al., Neutron-Antineutron Oscillations: A Snowmass 2013 White Paper. arXiv:1310.8593 [hep-ex]

41. K.S. Babu et al., Working Group Report: Baryon Number Violation. arXiv:1311.5285

42. A. Addazi, Z. Berezhiani, Y. Kamyshkov, Gauged $B-L$ number and neutron?antineutron oscillation: long-range forces mediated by baryophotons. Eur. Phys. J. C 77(5), 301 (2017). arXiv: 1607.00348

43. Z. Berezhiani, L. Bento, Fast neutron-mirror neutron oscillation and ultra high energy cosmic rays. Phys. Lett. B 635, 253 (2006). arXiv:hep-ph/0602227

44. Z. Berezhiani, A. Gazizov, Neutron oscillations to parallel world: earlier end to the cosmic ray spectrum? Eur. Phys. J. C 72, 2111 (2012). arXiv:1109.3725

45. R.N. Mohapatra, S. Nasri, S. Nussinov, Some implications of neutron mirror neutron oscillation. Phys. Lett. B 627, 124 (2005). arXiv:hep-ph/0508109

46. A. Coc, J.P. Uzan, E. Vangioni, Mirror matter can alleviate the cosmological lithium problem. Phys. Rev. D 87(12), 123530 (2013). arXiv: 1303.1935

47. Y.N. Pokotilovski, On the experimental search for neutronmirror neutron oscillations. Phys. Lett. B 639, 214 (2006). arXiv:nucl-ex/0601017

48. Z. Berezhiani, M. Frost, Y. Kamyshkov, B. Rybolt, L. Varriano, Neutron disappearance and regeneration from mirror state. Phys. Rev. D 96(3), 035039 (2017). arXiv:1703.06735

49. G. Ban et al., A direct experimental limit on neutron-mirror neutron oscillations. Phys. Rev. Lett. 99, 161603 (2007). arXiv:0705.2336

50. A. Serebrov et al., Experimental search for neutron-mirror neutron oscillations using storage of ultracold neutrons. Phys. Lett. B 663, 181 (2008). arXiv:0706.3600

51. I. Altarev et al., Neutron to mirror neutron oscillations in the presence of mirror magnetic fields. Phys. Rev. D 80, 032003 (2009). arXiv:0905.4208

52. K. Bodek et al., Additional results from the dedicated search for neutron mirror neutron oscillations. Nucl. Instrum. Meth. A 611, 141 (2009)

53. A. Serebrov et al., Search for neutron mirror neutron oscillations in a laboratory experiment with ultracold neutrons. Nucl. Instrum. Meth. A 611, 137 (2009). arXiv:0809.4902

54. Z. Berezhiani, R. Biondi, P. Geltenbort, I.A. Krasnoshchekova, V.E. Varlamov, A.V. Vassiljev, O.M. Zherebtsov, New experimental limits on neutron-mirror neutron oscillations in the presence of mirror magnetic field. Eur. Phys. J. C 78, 717 (2018). arXiv: 1712.05761

55. C. Abel et al. (nEDM Collaboration), A search for neutron to mirror-neutron oscillations. Phys. Lett. B 812, 135993 (2021). arXiv:2009.11046

56. Z. Berezhiani, F. Nesti, Magnetic anomaly in UCN trapping: signal for neutron oscillations to parallel world? Eur. Phys. J. C 72, 1974 (2012). arXiv:1203.1035

57. L.J. Broussard et al., New search for mirror neutron regeneration. EPJ Web Conf. 219, 07002 (2019). arXiv:1912.08264

58. A. Addazi et al., New high-sensitivity searches for neutrons converting into antineutrons and/or sterile neutrons HIBEAM/NNBAR experiment at the European Spallation Source. J. Phys. G 48(7), 070501 (2021). arXiv:2006.04907

59. N.J. Ayres et al., Improved search for neutron to mirror-neutron oscillations in the presence of mirror magnetic fields with a 
dedicated apparatus at the PSI UCN source. arXiv:2111.02794 [physics.ins-det]

60. Z. Berezhiani, Neutron lifetime puzzle and neutron-mirror neutron oscillation. Eur. Phys. J. C 79(6), 484 (2019). arXiv: 1807.07906

61. R.N. Mohapatra, S. Nussinov, Constraints on mirror models of dark matter from observable neutron-mirror neutron oscillation. Phys. Lett. B 776, 22 (2018). arXiv:1709.01637

62. Z. Berezhiani, Unusual effects in $n-n^{\prime}$ conversion, talk at the International Workshop INT-17-69W, Seattle, 23-27 Oct. (2017). http://www.int.washington.edu/talks/WorkShops/ int_17_69W/People/Berezhiani_Z/Berezhiani3.pdf

63. M. Mannarelli, Z. Berezhiani, R. Biondi, F. Tonelli, " $\mathrm{nn}$ ' conversion and neutron stars", talk at NORDITA Workshop "Particle Physics with Neutrons at the ESS", Stockholm, 10 14 Dec. (2018). https://indico.fysik.su.se/event/6570/timetable/\# 20181213

64. Z. Berezhiani, Neutron lifetime and dark decay of the neutron and hydrogen. LHEP 2(1), 118 (2019). arXiv:1812.11089

65. I. Goldman, R.N. Mohapatra, S. Nussinov, Bounds on neutronmirror neutron mixing from pulsar timing. Phys. Rev. D 100(12), 123021 (2019). arXiv: 1901.07077

66. I. Goldman, Astrophysical bounds on mirror dark matter derived from binary pulsars timing data. arXiv:2011.12070 [astro-ph.HE]

67. R. Ciancarella, F. Pannarale, A. Addazi, A. Marciano, Constraining mirror dark matter inside neutron stars. Phys. Dark Univ. 32, 100796 (2021). arXiv:2010.12904

68. J.M. Lattimer, The nuclear equation of state and neutron star masses. Annu. Rev. Nucl. Part. Sci. 62, 485 (2012). arXiv: 1305.3510

69. I. Vidana, A short walk through the physics of neutron stars. Eur. Phys. J. Plus 133(10), 445 (2018). arXiv: 1805.00837

70. Z. Berezhiani, A possible shortcut for neutron-antineutron oscillation through mirror world. Eur. Phys. J. C 81(1), 33 (2021). arXiv:2002.05609

71. R.C. Tolman, Static solutions of Einstein's field equations for spheres of fluid. Phys. Rev. 55, 364 (1939)

72. J.R. Oppenheimer, G.M. Volkoff, On massive neutron cores. Phys. Rev. 55, 374 (1939)

73. P. Demorest, T. Pennucci, S. Ransom, M. Roberts, J. Hessels, Shapiro delay measurement of a two solar mass neutron star. Nature 467, 1081 (2010). arXiv: 1010.5788

74. J. Antoniadis et al., A massive pulsar in a compact relativistic binary. Science 340, 6131 (2013). arXiv: 1304.6875

75. H.T. Cromartie et al. [NANOGrav Collaboration], Relativistic Shapiro delay measurements of an extremely massive millisecond pulsar. Nat. Astron. 4(1), 72 (2019). arXiv:1904.06759

76. P.C.C. Freire et al., Eight new millisecond pulsars in NGC 6440 and NGC 6441. Astrophys. J. 675, 670 (2008). arXiv:0711.0925

77. F. Douchin, P. Haensel, A unified equation of state of dense matter and neutron star structure. Astron. Astrophys. 380, 151 (2001). arXiv:astro-ph/0111092

78. F. Özel, P. Freire, Masses, radii, and the equation of state of neutron stars. Annu. Rev. Astron. Astrophys. 54, 401 (2016). arXiv: 1603.02698

79. G.F. Burgio, H.J. Schulze, I. Vidana, J.B. Wei, A modern view of the equation of state in nuclear and neutron star matter. Symmetry 13(3), 400 (2021)

80. H. Mueller, B.D. Serot, Relativistic mean field theory and the high density nuclear equation of state. Nucl. Phys. A 606, 508 (1996). arXiv:nucl-th/9603037

81. C.E. Rhoades Jr., R. Ruffini, Maximum mass of a neutron star. Phys. Rev. Lett. 32, 324 (1974)

82. H. Heiselberg, M. Hjorth-Jensen, Phases of dense matter in neutron stars. Phys. Rep. 328, 237-327 (2000). arXiv:nucl-th/9902033
83. A.R. Bodmer, Collapsed nuclei. Phys. Rev. D 4, 1601 (1971)

84. E. Witten, Cosmic separation of phases. Phys. Rev. D 30, 272 (1984)

85. Z. Berezhiani, I. Bombaci, A. Drago, F. Frontera, A. Lavagno, Gamma-ray bursts from delayed collapse of neutron stars to quark matter stars. Astrophys. J. 586, 1250 (2003). arXiv:astro-ph/0209257

86. Z. Berezhiani, I. Bombaci, A. Drago, F. Frontera, A. Lavagno, Gamma-ray bursts from delayed quark deconfinement phase transition in neutron stars. Nucl. Phys. Proc. Suppl. 113, 268 (2002)

87. A. Drago, A. Lavagno, G. Pagliara, Effects of color superconductivity on the structure and formation of compact stars. Phys. Rev. D 69, 057505 (2004). arXiv:nucl-th/0401052

88. M. Alford, M. Braby, M.W. Paris, S. Reddy, Hybrid stars that masquerade as neutron stars. Astrophys. J. 629, 969-978 (2005). arXiv:nucl-th/0411016

89. I. Bombaci, A. Drago, D. Logoteta, G. Pagliara, I. Vidaña, Was GW190814 a black hole-strange quark star system? Phys. Rev. Lett. 126(16), 162702 (2021). arXiv:2010.01509

90. A. Drago, G. Pagliara, Combustion of a hadronic star into a quark star: the turbulent and the diffusive regimes. Phys. Rev. C 92(4), 045801 (2015). arXiv: 1506.08337

91. A. Drago, G. Pagliara, The scenario of two families of compact stars: 2 . Transition from hadronic to quark matter and explosive phenomena. Eur. Phys. J. A 52(2), 41 (2016). arXiv:1509.02134

92. A. Drago, A. Lavagno, G. Pagliara, Can very compact and very massive neutron stars both exist? Phys. Rev. D 89(4), 043014 (2014). arXiv:1309.7263

93. I. Bombaci, D. Logoteta, Equation of state of dense nuclear matter and neutron star structure from nuclear chiral interactions. Astron. Astrophys. 609, A128 (2018). arXiv: 1805.11846

94. D. Logoteta, I. Vidana, I. Bombaci, Impact of chiral hyperonic three-body forces on neutron stars. Eur. Phys. J. A 55(11), 207 (2019). arXiv:1906.11722

95. A. Drago, A. Lavagno, G. Pagliara, D. Pigato, Early appearance of $\Delta$ isobars in neutron stars. Phys. Rev. C 90(6), 065809 (2014). arXiv: 1407.2843

96. G. Dvali, M. Redi, Phenomenology of $10^{32}$ dark sectors. Phys. Rev. D 80, 055001 (2009). arXiv:0905.1709

97. Z.G. Berezhiani, M.I. Vysotsky, Neutrino decay in matter. Phys. Lett. B 199, 281 (1987)

98. Z.G. Berezhiani, A.Y. Smirnov, Matter induced neutrino decay and supernova SN1987A. Phys. Lett. B 220, 279 (1989)

99. Z. Berezhiani, R. Biondi, Y. Kamyshkov, L. Varriano, On the neutron transition magnetic moment. MDPI Phys. 1(2), 271 (2019). arXiv: 1812.11141

100. Z. Berezhiani, A. Vainshtein, Neutron-antineutron oscillation and discrete symmetries. Int. J. Mod. Phys. A 33(31), 1844016 (2018)

101. Z. Berezhiani, A. Vainshtein, Neutron-antineutron oscillations: discrete symmetries and quark operators. Phys. Lett. B 788, 58 (2019). arXiv:1809.00997

102. J.P.W. Verbiest et al., Precision timing of PSR J0437-4715: an accurate pulsar distance, a high pulsar mass and a limit on the variation of Newton's gravitational constant. Astrophys. J. 679, 675 (2008). arXiv:0801.2589

103. J.M. Weisberg, D.J. Nice, J.H. Taylor, Timing measurements of the relativistic binary pulsar PSR B1913+16. Astrophys. J. 722, 1030 (2010). arXiv:1011.0718

104. M. Durant et al., The spectrum of the recycled PSR J0437-4715 and its white dwarf companion. Astrophys. J. 746, 6 (2012). arXiv: 1111.2346

105. B. Rangelov et al., Hubble space telescope detection of the millisecond pulsar J2124-3358 and its far-ultraviolet bow shock nebula. Astrophys. J. 835(2), 264 (2017). arXiv:1701.00002

106. G.G. Pavlov et al., Old but still warm: far-UV detection of PSR B0950+08. Astrophys. J. 850(1), 79 (2017). arXiv: 1710.06448 
107. S. Guillot et al., Hubble space telescope nondetection of PSR J2144-3933: the coldest known neutron star. Astrophys. J. 874(2), 175 (2019). arXiv: 1901.07998

108. M.G. Alford, A. Schmitt, K. Rajagopal, T. Schäfer, Color superconductivity in dense quark matter. Rev. Mod. Phys. 80, 14551515 (2008). arXiv:0709.4635

109. R. Anglani, R. Casalbuoni, M. Ciminale, N. Ippolito, R. Gatto, M. Mannarelli, M. Ruggieri, Crystalline color superconductors. Rev. Mod. Phys. 86, 509-561 (2014). arXiv:1302.4264

110. A. Drago, G. Pagliara, Why can hadronic stars convert into strange quark stars with larger radii. Phys. Rev. D 102(6), 063003 (2020). arXiv:2007.03436

111. F. Ozel, D. Psaltis, Z. Arzoumanian, S. Morsink, M. Baubock, Measuring neutron star radii via pulse profile modeling with NICER. Astrophys. J. 832(1), 92 (2016). arXiv:1512.03067

112. R. Cerulli et al., DAMA annual modulation and mirror dark matter. Eur. Phys. J. C 77(2), 83 (2017). arXiv:1701.08590

113. A. Addazi et al., DAMA annual modulation effect and asymmetric mirror matter. Eur. Phys. J. C 75(8), 400 (2015). arXiv:1507.04317

114. Z. Berezhiani, A. Lepidi, Cosmological bounds on the "millicharges" of mirror particles. Phys. Lett. B 681, 276 (2009). arXiv:0810.1317

115. C. Vigo, L. Gerchow, B. Radics, M. Raaijmakers, A. Rubbia, P. Crivelli, Phys. Rev. Lett. 124(10), 101803 (2020). arXiv: 1905.09128

116. Z. Berezhiani, A.D. Dolgov, I.I. Tkachev, Dark matter and generation of galactic magnetic fields. Eur. Phys. J. C 73, 2620 (2013). arXiv: 1307.6953

117. B.P. Abbott et al. [LIGO Scientific and Virgo Collaborations], GW170817: observation of gravitational waves from a binary neutron star inspiral. Phys. Rev. Lett. 119(16), 161101 (2017). arXiv: 1710.05832

118. B.P. Abbott et al. [LIGO Scientific and Virgo Collaborations], GW190425: observation of a compact binary coalescence with total mass $\sim 3.4 M_{\odot}$. Astrophys. J. Lett. 892(1), L3 (2020). arXiv:2001.01761

119. A. Addazi, A. Marcianò, Testing merging of dark exotic stars from gravitational waves in the multi-messenger approach. Int. J. Mod. Phys. A 33(29), 1850167 (2018). arXiv:1710.08822
120. R. Beradze, M. Gogberashvili, A.S. Sakharov, Binary neutron star mergers with missing electromagnetic counterparts as manifestations of mirror world. Phys. Lett. B 804, 135402 (2020). arXiv: 1910.04567

121. R. Beradze, M. Gogberashvili, Unexpected LIGO events and the Mirror World. Mon. Not. R. Astron. Soc. 503, 2882 (2021). arXiv:2101.12532

122. B.P. Abbott et al. [LIGO Scientific and Virgo Collaborations], GW170817: measurements of neutron star radii and equation of state. Phys. Rev. Lett. 121(16), 161101 (2018). arXiv:1805.11581

123. G.F. Burgio, A. Drago, G. Pagliara, H.-J. Schulze, J.-B. Wei, Are small radii of compact stars ruled out by GW170817/AT2017gfo? Astrophys. J. 860(2), 139 (2018). arXiv:1803.09696

124. Z. Berezhiani, Antistars or antimatter cores in mirror neutron stars?. arXiv:2106.11203 [astro-ph.HE]

125. Z. Berezhiani, Anti-dark matter: a hidden face of mirror world. arXiv:1602.08599 [astro-ph.CO]

126. F. Sandin, P. Ciarcelluti, Effects of mirror dark matter on neutron stars. Astropart. Phys. 32, 278 (2009). arXiv:0809.2942

127. I. Goldman, R.N. Mohapatra, S. Nussinov, D. Rosenbaum, V. Teplitz, Possible implications of asymmetric fermionic dark matter for neutron stars. Phys. Lett. B 725, 200 (2013). arXiv: 1305.6908

128. D. McKeen, A.E. Nelson, S. Reddy, D. Zhou, Neutron stars exclude light dark baryons. Phys. Rev. Lett. 121(6), 061802 (2018). arXiv: 1802.08244

129. G. Baym, D.H. Beck, P. Geltenbort, J. Shelton, Testing dark decays of baryons in neutron stars. Phys. Rev. Lett. 121(6), 061801 (2018). arXiv: 1802.08282

130. J.M. Cline, J.M. Cornell, Dark decay of the neutron. JHEP 1807, 081 (2018). arXiv:1803.04961

131. G. Narain, J. Schaffner-Bielich, I.N. Mishustin, Compact stars made of fermionic dark matter. Phys. Rev. D 74, 063003 (2006). arXiv:astro-ph/0605724 